Research Paper

\title{
Clinical significance of long non-coding RNA DUXAP8 and its protein coding genes in hepatocellular carcinoma
}

\author{
Xiang-Kun Wang1, Xi-Wen Liao1, Rui Huang², Jian-Lu Huang,1,3, Zi-Jun Chen'1, Xin Zhou ${ }^{1}$, Cheng-Kun \\ Yang1, Chuang-Ye Han ${ }^{1}$, Guang-Zhi Zhu1, Tao Peng ${ }^{1 凶}$ \\ 1. Department of Hepatobiliary Surgery, The First Affiliated Hospital of Guangxi Medical University, Nanning, 530021, Guangxi Province, China; \\ 2. Department of Hematology, The First Affiliated Hospital of Guangxi Medical University, Nanning, 530021, Guangxi Province, People's Republic of China; \\ 3. Department of Hepatobiliary Surgery, The Third Affiliated Hospital of Guangxi Medical University, Nanning 530031, Guangxi Province, China. \\ $\triangle$ Corresponding author: Tao Peng, M.D., Professor, Director, Department of Hepatobiliary Surgery, the First Affiliated Hospital of Guangxi \\ Medical University, Nanning, 530021, Guangxi Province, China. Tel: (+86)-771-5356528. Fax: (+86)-771-5350031. E-mail: pengtaogmu@163.com.
}

(C) The author(s). This is an open access article distributed under the terms of the Creative Commons Attribution License (https://creativecommons.org/licenses/by/4.0/). See http://ivyspring.com/terms for full terms and conditions.

Received: 2020.05.07; Accepted: 2020.08.16; Published: 2020.08.25

\begin{abstract}
Backgrounds: Hepatocellular carcinoma $(\mathrm{HCC})$ is a lethal malignancy worldwide that is difficult to diagnose during the early stages and its tumors are recurrent. Long non-coding RNAs (IncRNAs) have increasingly been associated with tumor biomarkers for diagnosis and prognosis. This study attempts to explore the potential clinical significance of IncRNA DUXAP8 and its co-expression related protein coding genes (PCGs) for HCC.

Method: Data from a total of $370 \mathrm{HCC}$ patients from The Cancer Genome Atlas were utilized for the analysis. DUXAP8 and its top 10 PCGs were explored for their diagnostic and prognostic implications for HCC. A risk score model and nomogram were constructed for prognosis prediction using prognosis-related genes and DUXAP8. Molecular mechanisms of DUXAP8 and its PCGs involved in HCC initiation and progression were investigated. Then, potential target drugs were identified using genome-wide DUXAP8-related differentially expressed genes in a Connectivity Map database.

Results: The top 10 PCGs were identified as: RNF2, MAGEA1, GABRA3, MKRN3, FAMI33A, MAGEA3, CNTNAP4, MAGEA6, MALRDI, and DGKI. Diagnostic analysis indicated that DUXAP8, MEGEAI, MKRN3, and $D G K I$ show diagnostic implications (all area under curves $\geq 0.7, p \leq 0.05$ ). Prognostic analysis indicated that DUXAP8 and RNF2 had prognostic implications for HCC (adjusted $p=0.014$ and 0.008 , respectively). The risk score model and nomogram showed an advantage for prognosis prediction. A total of 3 target drugs were determined: cinchonine, bumetanide and amiprilose and they may serve as potential therapeutic targets for HCC.

Conclusion: Functioning as an oncogene, DUXAP8 is overexpressed in tumor tissue and may serve as both a diagnostic and prognosis biomarker for HCC. MEGEAI, MKRN3, and DGKI maybe potential diagnostic biomarkers and DGKI may also be potentially prognostic biomarkers for HCC.
\end{abstract}

Key words: long non-coding RNA; DUXAP8; protein-coding gene; hepatocellular carcinoma; molecular mechanism

\section{Introduction}

By 2018 , it was estimated that nearly 841,800 new liver cancer cases and 781,631 deaths due to liver cancers were recorded across 20 world regions (1). Hepatocellular carcinoma (HCC) accounts for approximately $80 \%$ of primary liver cancers, and ranks fifth among the most common malignancies and the third leading cause of cancer-related mortality worldwide $(2,3)$. Hepatitis B or C (HBV) viral infections are known to cause chronic liver cirrhosis and account for approximately $80-90 \%$ of all the HCC cases (4). Other risk factors include excessive alcohol consumption, obesity, aflatoxin B contamination, iron overload, and environmental pollutants $(5,6)$. HCC is a fatal malignancy characterized by high metastasis and recurrence after surgery leading to poor prognosis and very low survival rates $(7,8)$. Several treatments including hepatic resection, liver transplantation, image-guided tumor ablation, 
transcatheter tumor therapy, and systemic therapy have been developed to control HCC (9). However, patient survival rates are still low despite these treatments. The 5-year survival rate of HCC is about $7 \%$ (10). Identification of novel diagnostic biomarkers and therapeutic targets is crucial for improving prognosis of HCC patients.

Long non-coding RNAs (lncRNAs) regulate normal cellular functions $(11,12)$. They also participate in various biological and pathological processes such as tumorigenesis (13). Overexpression of CCAT2 suppresses cell migration, invasion, and growth, and induce early apoptosis of glioma cells (14). LncRNAs have been reported to regulate pathogenesis of HCC (15). For instance, HULC (16) and LINC00974 (17) have been reported to participate in HCC development and progression. LINC00673 regulates HCC carcinogenesis via microRNA-205 and is upregulated in HCC tissues and cell lines (12).

DUXAP8, a pseudogene derived from lncRNA, promotes growth of pancreatic carcinoma cells by epigenetically silencing CDKN1A and KLF2 (18). It has also been found to enhance renal cell carcinoma by downregulating microRNA-126 (19). DUXAP8 is differentially expressed in bladder cancer, its downregulation inhibits cell invasion and proliferation and leads to cell apoptosis (20). Lijuan et al. reported that DUXAP8 expression was positively correlated with lymph node metastasis and clinical stage in esophageal cancer (21). This suggested that DUXAP8 is up-regulated and increases metastasis of renal cell carcinoma cells (22). DUXAP8 knockdown resulted in clear cell cycle arrest at the G0/G1 phase (23). This showed that DUXAP8 may be an effective prognostic biomarker and treatment target in non-small cell lung cancer (23). Genome-wide analysis showed that DUXAP8 was not only differentially expressed in esophageal cancer but was a diagnostic and therapeutic target for cancer (24). Despite these studies, little is known about the association between DUXAP8 and HCC. Therefore, we assessed the diagnostic and prognostic implications, molecular mechanism and potential target drugs of DUXAP8 in HCC.

\section{Materials and Methods}

\section{Data processing and identification of protein-coding gene (PCG)}

HCC patient expression data was downloaded from The Cancer Genome Atlas database (TCGA, https://cancergenome.nih.gov/). Criteria of patient selection as following: patients were pathologically diagnosed primary HCC, vital data including HBV infection status, alcohol history, vascular invasion and radical resection should be collected, all the patients have definite alive/die status and survival time. LncRNAs function together with their co-expression-related PCGs and co-expression coefficient. Therefore, evaluated to acquire its PCGs was conducted using R 3.5.0 (https://www.rproject.org/) $(25,26)$. The top 10 PCGs and DUXAP8 underwent further analysis. In addition, low and high expressions of DUXAP8 and the top 10 PCGs were used as cut-off median expressions of DUXAP8.

\section{Body distribution, tumor, and non-tumor expression}

Body distribution of DUXAP8 and its top 10 PCGs were obtained from the Gene Expression Profiling Interactive Analysis database (GEPIA, http://gepia.cancer-pku.cn/index.html) (27). Scatter plots of the tumor and non-tumor tissues were plotted using Graphpad 7.0.

\section{Determination of diagnostic and prognostic significance of DUXAP8 and its PCGs}

Diagnostic Receiver Operating Characteristic (ROC) curves of DUXAP8 and the PCGs were depicted using data from tumor and non-tumor tissue expressions using Graphpad 7.0. An Area Under Curve (AUC) value $\geq 0.7$ represented high diagnostic significance. The joint-effect analysis was performed using the diagnosis-related lncRNA and genes.

In addition, prognostic significance was calculated and visualized using Kaplan-Meier plots. Multivariate analysis was performed using prognosis-related clinical factors. The joint-effect analysis was also performed using prognosis-related lncRNA and genes.

\section{Construction of the risk score model and nomogram}

A risk score model and nomogram were constructed for HCC prognosis prediction. Risk scores, patient survival status, DUXAP8 gene expression heat maps, Kaplan-Meier plot, and time-dependent ROC curves were entered into the model. The risk score was formulated as follows: risk score $=$ expression of gene $1 \times \beta_{1}$ of gene ${ }_{1}+$ expression of gene $2 \times \beta_{2}$ of gene ${ }_{2}+\ldots+$ expression of gene ${ }_{n} \times \beta_{n}$ of gene $_{n}(28,29)$. Contribution coefficients $(\beta)$ were obtained from the multivariate Cox proportional hazards regression model.

The nomogram was constructed using prognosis-related genes, DUXAP8, and clinical factors. Differential expressions and clinical factors indicate varied points. The 1, 3-, and 5- year overall survivals (OS) were included in the nomogram. OS prediction at 1,3, and 5 year can be found accordingly 
from the points.

\section{Gene set enrichment analysis (GSEA)}

GSEA was performed to assess molecular mechanisms of DUXAP8 and the PCGs. Gene ontologies (GOs), including biological processes (BP, c5.bp.v6.1.symbols.gmt,), cellular components (CC, c5.cc.v6.1.symbols.gmt), molecular functions (MF, c5.mf.v6.1.symbols.gmt), and Kyoto Encyclopedia of Genes and Genomes (KEGG, c2.cp.kegg.v6.1. symbols.gmt) pathway sets were used to find the biological processes associated with DUXAP8 and its PCGs.

\section{Co-expression matrix, gene-gene interaction (GGI) and protein-protein interaction (PPI) network}

The co-expression matrix for DUXAP8 and its PCGs of Pearson correlation were constructed using R 3.5.0. Different colors indicate either positive or negative correlations. The GGI network was constructed and their co-expression relationship with other genes was determined using the geneMANIA plugin of Cytoscape software $(30,31)$. The PPI network was constructed using the STRING database (https://string-db.org/) (32). Co-expression and co-occurrence interactions are presented using STRING.

\section{Pharmacological target drug identification and enrichment analysis}

Genome-wide differentially expressed genes (DEGs) in volcano plots and heatmaps were determined and plotted using the edgeR method (33). Results with a |Fold changel of $\geq 2$ and a p-value of $\leq 0.5$ were used for further analysis. Target drugs were then acquired using these DEGs obtained from the Connectivity Map database (https://portals. broadinstitute.org/cmap/). Positively correlated drugs were considered as potential targets. The 2D and 3D structure of the target drugs were obtained from PubChem Compound (https://www.ncbi.nlm. nih.gov/pccompound/).

Enriched metabolic pathways and biological processes were analyzed using the Database for Annotation, Visualization and Integrated Discovery (DAVID, https://david.ncifcrf.gov/) (34, 35). Visualization of GO was performed using the BinGO plugin of Cytoscape software (36).

\section{Validations of PCGs in public databases}

For further confirming potential values of these PCGs in HCC, we further used HCCDB (37) (http://lifeome.net/database/hccdb/home.html) and oncomine $(38,39)$ (https://www.oncomine.org/ resource/main.html) databases to confirm our results.

\section{Statistical analysis}

Survival and statistical analyses were performed using SPSS 16.0 (IBM, Chicago, IL). Median survival time, log-rank p-value, adjusted p-value, 95\% confidence interval (CI) and hazard ratio (HR) were calculated using the Kaplan-Meier method and Cox proportional hazards regression model. A p-value of $\leq 0.05$ was statistically significant.

\section{Results}

\section{Demographic characteristics and co-expression-related PCGs of DUXAP8}

A total of $370 \mathrm{HCC}$ patients were enrolled in this study. Clinical factors found to be linked with HCC were $\mathrm{HBV}$ infection, radical resection, and tumor stage OS $(\mathrm{p}<0.0001,=0.007$, and $<0.0001$, respectively). Detailed demographic characteristics are shown in Supplementary Table 1. Co-expression-related PCGs were identified and are shown in Supplementary Table 2. The top 10 PCGs were; RNF2, MAGEA1, GABRA3, MKRN3, FAM133A, MAGEA3, CNTNAP4, MAGEA6, MALRD1, and DGKI (Table 1). Pearson correlation coefficient $\mathrm{R}$ was used to evaluate the correlation between these PCGs and DUXAP8. PCGs with an $|R|$ value of $\geq 0.2$ were visualized in the network (Figure 7B).

Table 1. Top 10 protein-coding genes with DUXAP8

\begin{tabular}{lllll}
\hline LncRNA & PCG & Correlation & $95 \%$ CI & P value \\
\hline DUXAP8 & RNF2 & 0.43 & $0.35-0.51$ & $1.90 \mathrm{E}-18$ \\
DUXAP8 & MAGEA1 & 0.42 & $0.33-0.5$ & $4.86 \mathrm{E}-17$ \\
DUXAP8 & GABRA3 & 0.41 & $0.32-0.49$ & $4.55 \mathrm{E}-16$ \\
DUXAP8 & MKRN3 & 0.39 & $0.30-0.47$ & $8.87 \mathrm{E}-15$ \\
DUXAP8 & FAM133A & 0.38 & $0.29-0.47$ & $2.30 \mathrm{E}-14$ \\
DUXAP8 & MAGEA3 & 0.38 & $0.29-0.46$ & $4.70 \mathrm{E}-14$ \\
DUXAP8 & CNTNAP4 & 0.36 & $0.27-0.44$ & $1.38 \mathrm{E}-12$ \\
DUXAP8 & MAGEA6 & 0.36 & $0.26-0.44$ & $1.53 \mathrm{E}-12$ \\
DUXAP8 & MALRD1 & 0.36 & $0.26-0.44$ & $1.84 \mathrm{E}-12$ \\
DUXAP8 & DGKI & 0.35 & $0.26-0.44$ & $3.35 \mathrm{E}-12$ \\
\hline
\end{tabular}

Note: PCG: protein-coding gene, CI: confidence interval.

\section{Body maps, expression of DUXAP8 and the top 10 PCGs}

Body maps of DUXAP8 and the top 10 PCGs showed that DUXAP8 and RNF2 were highly expressed in liver in comparison to other PCGs (Figure 1). In comparison, DUXAP8 and RNF2 were highly expressed in kidney, lungs, and intestines relative to other PCGs. Except MALRD1, DUXAP8 and the other 9 PCGs were differentially expressed between tumor and non-tumor tissues (Figure 2). All PCGs and DUXAP8 were overexpressed in tumor tissues. 
A

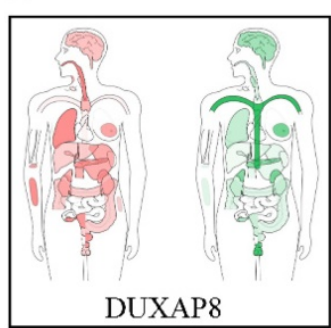

E
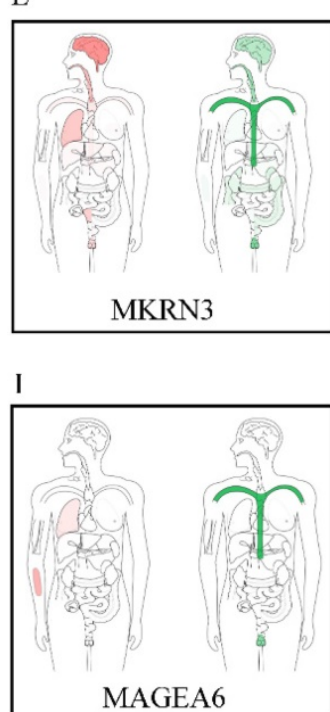

B

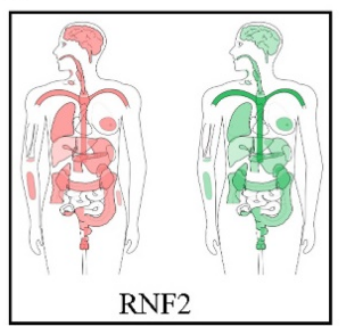

$\mathrm{F}$
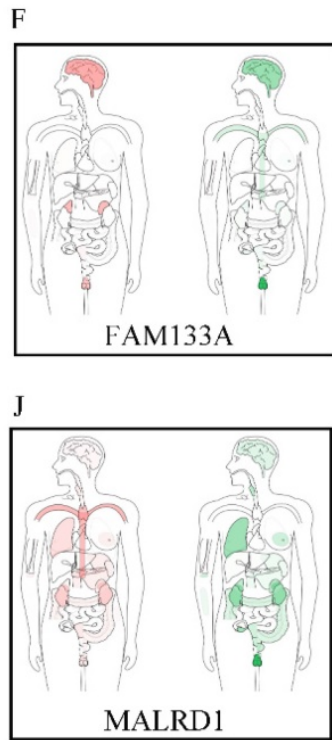

$\mathrm{C}$

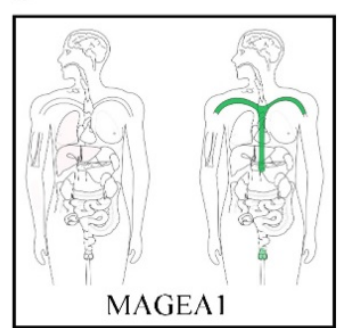

G

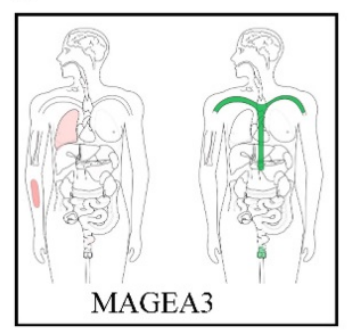

K

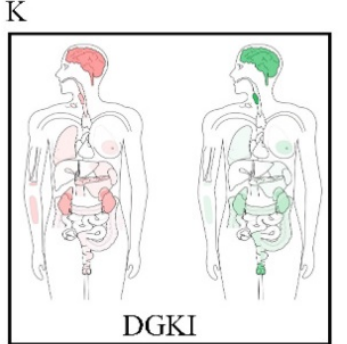

D

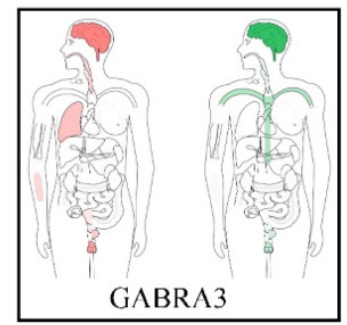

$\mathrm{H}$

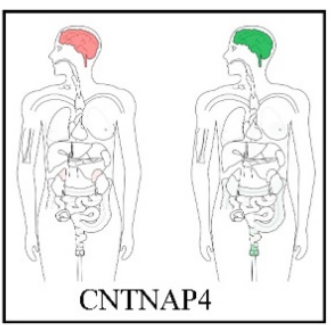

Tumor

Normal

Figure 1. Body map of the expressions of DUXAP8 and its co-expression-related protein-coding genes. A-K: Body map of the expressions of DUXAP8, RNF2, MAGEAI, GABRA3, MKRN3, FAMI33A, MAGEA3, CNTNAP4, MAGEA6, MALRDI, and DGKI, respectively.
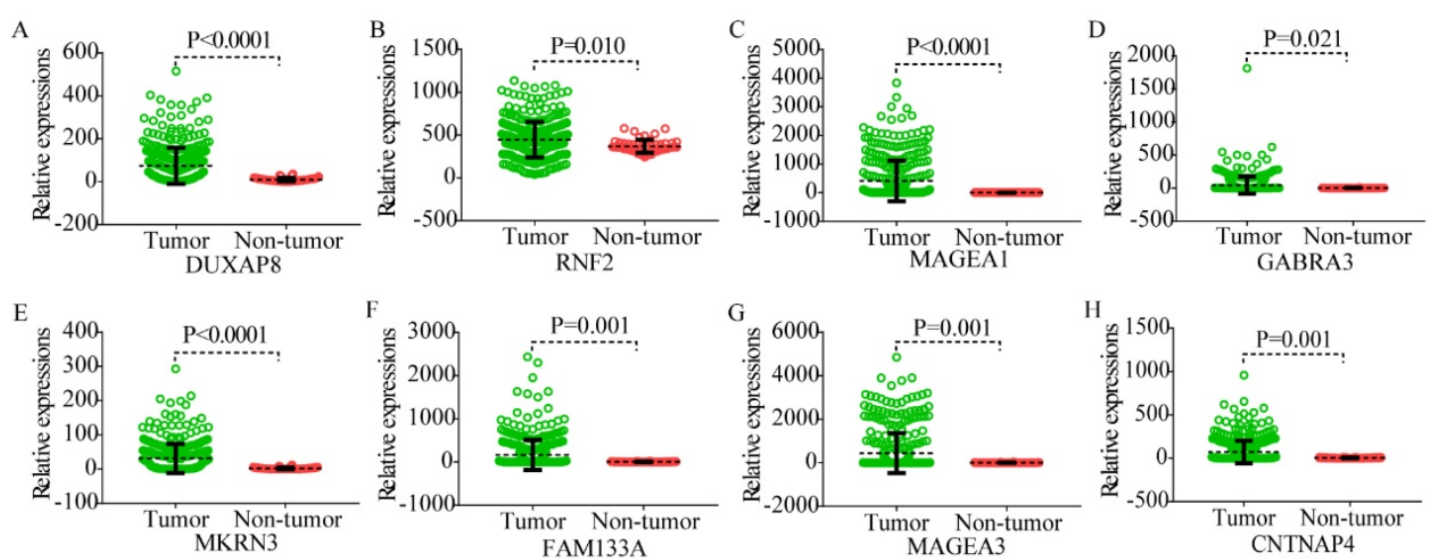

GABRA3
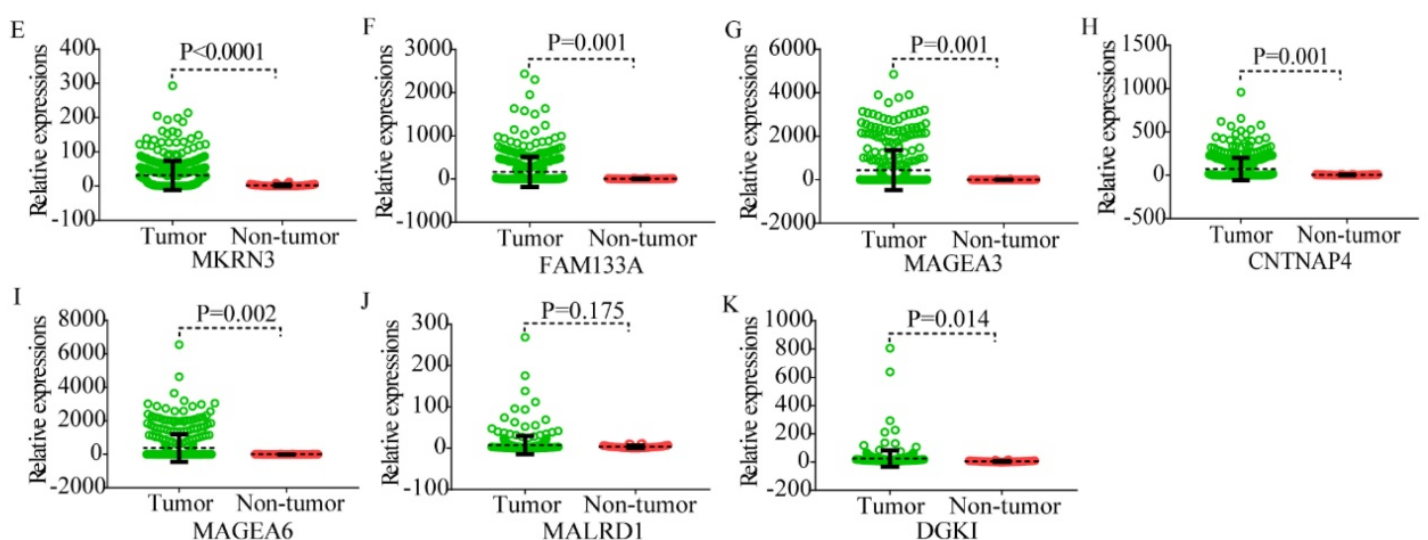

Figure 2. Scatter plots of DUXAP8 and its co-expression-related protein-coding genes in tumor and non-tumor tissues. A-K: Scatter plots of DUXAP8, RNF2, MAGEAI, GABRA3, MKRN3, FAMI33A, MAGEA3, CNTNAP4, MAGEA6, MALRDI, and DGKI, respectively.

\section{Diagnosis, prognosis and joint-effect analysis of DUXAP8 and the top 10 PCGs}

In the diagnosis analysis, DUXAP8, MEGEA1, $M K R N 3$, and DGKI were found to be potential diagnostic markers (AUC $=0.798,0.805,0.763$ and 0.905 , respectively; $\mathrm{p}<0.0001,<0.0001,<0.0001$, $=0.0001$ and $<0.0001$, respectively, (Figure 3). Other PCGs showed no diagnostic significance (all 
AUC <0.7). The joint-effect analysis of DUXAP8, MEGEA1, MKRN3, and DGKI indicated that their joint effect provided better diagnostic significance than when applied individually (AUC $=0.847,0.817,0.947$, 0.961 and 0.946, respectively; all $\mathrm{p}<0.0001$; Figure 4), except for MAGEA1 and MKRN3. The AUC value of this combination was equal to that of MAGEA1 alone (both AUC=0.805, 95\%CI=0.760-0.850; Figure 3C, 4D).

In the prognostic analysis, DUXAP8, RNF2, and GABRA3 showed prognostic value in univariate analysis (crude $\mathrm{p}=0.001,0.003$, and 0.006 , respectively; Figure 5, Table 2). However, only DUXAP8 and RNF2 showed prognostic value in multivariate analysis, after adjustment for tumor stage, $\mathrm{HBV}$ infection, and radical resection factors (adjusted $\mathrm{p}=0.014$ and 0.008 , respectively; Table 2). Also, combination of DUXAP8 and RNF2 showed better prognostic value than individual effects (crude $\mathrm{p}<0.0001$; adjusted $\mathrm{p}=0.001$; Figure 5L, Table 3).
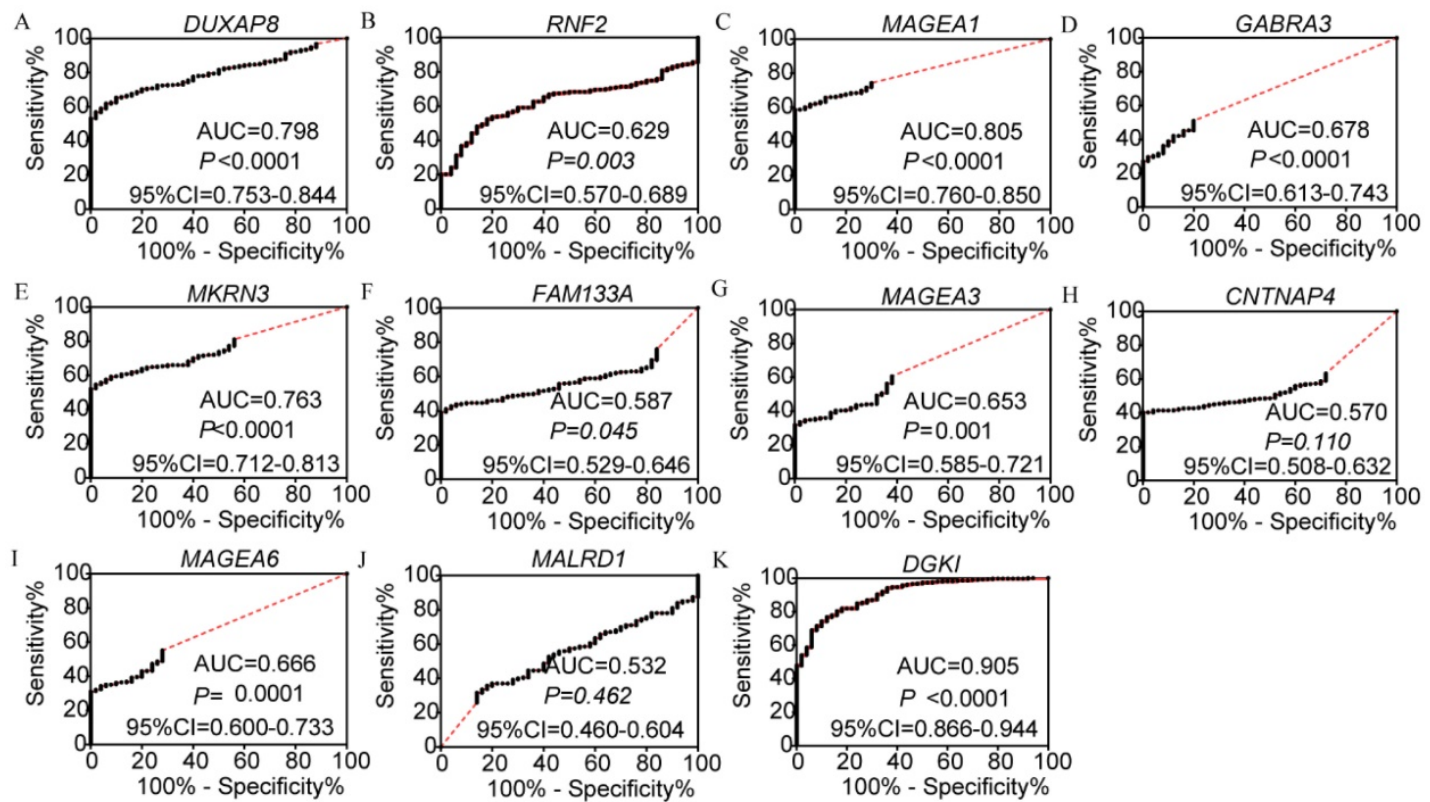

\section{Construction of risk score model and nomogram}

A risk score model was constructed using risk scores, patient survival status, and expressions levels DUXAP8 and RNF2 (Figure 6A, Table 4). low DUXAP8 and RNF2 expressions were associated with low risk and hence better prognosis (Figure 6A-B, Table 5). Time-dependent ROC curves suggested that the risk score model could predict the 1 and 3 years survival rate $(\mathrm{AUC}=0.705$ and 0.644 , respectively; Figure 6C). A nomogram was constructed using tumor stage, HBV infection status, radical resection status, and expressions of DUXAP8 and RNF2 (Figure 7A). Low expressions of DUXAP8 and RNF2, tumor stage I, without HBV infection and radical resection, were correlated with low-risk scores, and hence better patient survival.

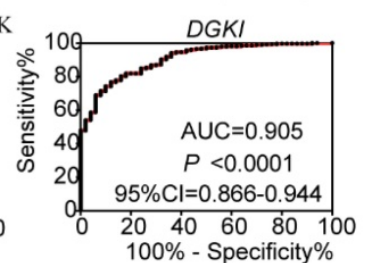

Figure 3. Diagnostic receiver operator curves of DUXAP8 and its co-expression-related protein-coding genes. A-K: Diagnostic receiver operator curves of DUXAP8, RNF2, MAGEAI, GABRA3, MKRN3, FAMI33A, MAGEA3, CNTNAP4, MAGEA6, MALRDI, and DGKI, respectively.
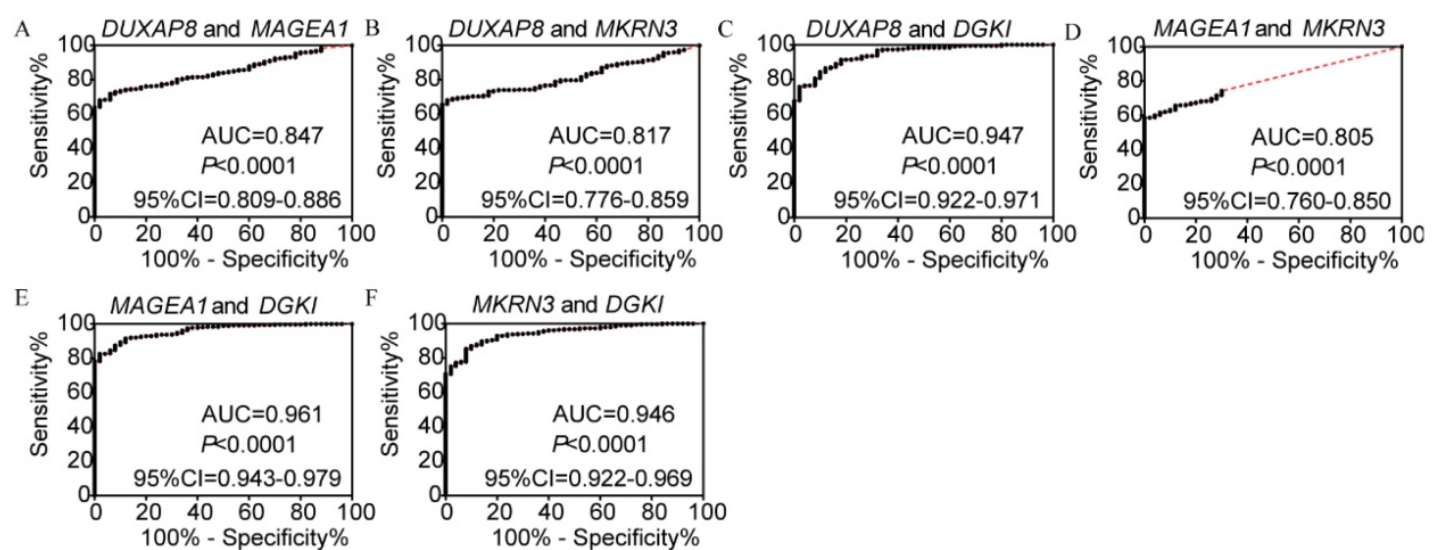

Figure 4. Joint-effect analysis of diagnostic receiver operator curves of DUXAP8 and diagnosis-related genes. A-F: Diagnostic receiver operator curves of DUXAP8 and MAGEAI; DUXAP8 and MKRN3; DUXAP8 and DGKI; MAGEAI and MKRN3; MAGEAI and DGKI; and MKRN3 and DGKI, respectively. 

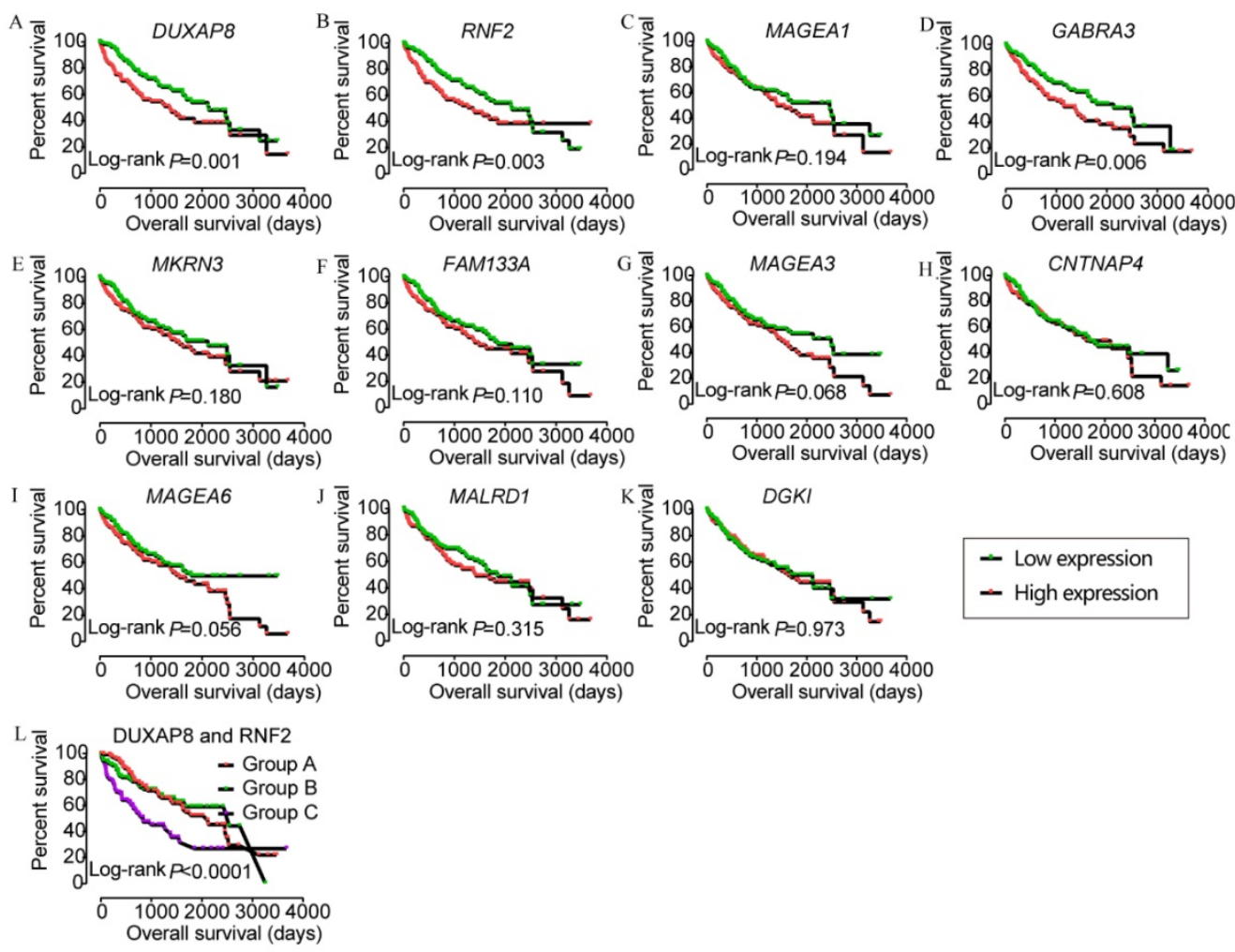

Figure 5. Kaplan-Meier plots of DUXAP8 and its co-expression-related protein-coding genes and joint-effect analysis of DUXAP8 and RNF2. A-L: Kaplan-Meier plots of DUXAP8, RNF2, MAGEAI, GABRA3, MKRN3, FAMI33A, MAGEA3, CNTNAP4, MAGEA6, MALRDI, DGKI, and joint-effect analysis plot of DUXAP8 and RNF2, respectively.

Table 2. Prognostic analysis of DUXAP8 and target genes for overall survival

\begin{tabular}{|c|c|c|c|c|c|c|c|}
\hline \multirow[t]{2}{*}{ Long non-coding RNA } & \multirow{2}{*}{$\frac{\text { Patients }}{(\mathrm{n}=370)}$} & \multicolumn{6}{|c|}{ Overall survival } \\
\hline & & No. of event & MST (days) & $\mathrm{HR}(95 \% \mathrm{CI})$ & Crude $P$ value & $\mathrm{HR}(95 \% \mathrm{CI})$ & Adjusted $P$ value \\
\hline \multicolumn{8}{|l|}{ DUXAP8 } \\
\hline Low expression & 185 & 56 & 2131 & Ref. & 0.001 & Ref. & 0.014 \\
\hline $\begin{array}{l}\text { High expression } \\
\text { RNF2 }\end{array}$ & 185 & 74 & 1372 & $1.768(1.247-2.507)$ & & $1.632(1.104-2.412)$ & \\
\hline Low expression & 185 & 57 & 2131 & Ref. & 0.003 & Ref. & 0.008 \\
\hline $\begin{array}{l}\text { High expression } \\
\text { MAGEA1 }\end{array}$ & 185 & 73 & 1372 & $1.704(1.199-2.420)$ & & $1.700(1.146-2.519)$ & \\
\hline Low expression & 185 & 59 & 2456 & Ref. & 0.194 & Ref. & 0.480 \\
\hline $\begin{array}{l}\text { High expression } \\
\text { GABRA3 }\end{array}$ & 185 & 71 & 1397 & $1.258(0.889-1.780)$ & & $1.147(0.784-1.678)$ & \\
\hline Low expression & 185 & 55 & 2486 & Ref. & 0.006 & Ref. & 0.103 \\
\hline $\begin{array}{l}\text { High expression } \\
\text { MKRN3 }\end{array}$ & 185 & 75 & 1372 & $1.631(1.149-2.313)$ & & $1.378(0.937-2.026)$ & \\
\hline Low expression & 185 & 58 & 2116 & Ref. & 0.180 & Ref. & 0.672 \\
\hline $\begin{array}{l}\text { High expression } \\
\text { FAM133A }\end{array}$ & 185 & 72 & 1560 & $1.267(0.896-1.792)$ & & $1.086(0.740-1.595)$ & \\
\hline Low expression & 185 & 61 & 1852 & Ref. & 0.110 & Ref. & 0.472 \\
\hline $\begin{array}{l}\text { High expression } \\
M A G E A 3\end{array}$ & 185 & 69 & 1397 & $1.325(0.938-1.872)$ & & $1.152(0.784-1.694)$ & \\
\hline Low expression & 185 & 57 & 2486 & Ref. & 0.068 & Ref. & 0.207 \\
\hline $\begin{array}{l}\text { High expression } \\
\text { CNTNAP4 }\end{array}$ & 185 & 73 & 1490 & $1.381(0.976-1.955)$ & & $1.280(0.872-1.878)$ & \\
\hline Low expression & 185 & 65 & 1694 & Ref. & 0.608 & Ref. & 0.952 \\
\hline $\begin{array}{l}\text { High expression } \\
M A G E A 6\end{array}$ & 185 & 65 & 1624 & $1.094(0.776-1.544)$ & & $0.988(0.675-1.446)$ & \\
\hline Low expression & 185 & 56 & 1791 & Ref. & 0.056 & Ref. & 0.186 \\
\hline $\begin{array}{l}\text { High expression } \\
\text { MALRD1 }\end{array}$ & 185 & 74 & 1560 & $1.404(0.992-1.988)$ & & $1.298(0.882-1.909)$ & \\
\hline Low expression & 185 & 61 & 1852 & Ref. & 0.315 & Ref. & 0.707 \\
\hline $\begin{array}{l}\text { High expression } \\
\text { DGKI }\end{array}$ & 185 & 69 & 1423 & $1.194(0.845-1.686)$ & & $1.076(0.734-1.577)$ & \\
\hline Low expression & 185 & 61 & 2116 & Ref. & 0.973 & Ref. & 0.170 \\
\hline High expression & 185 & 69 & 1624 & $1.006(0.712-1.421)$ & & $0.763(0.518-1.123)$ & \\
\hline
\end{tabular}

Note: §: P values were adjusted for radical resection, tumor stage and HBV infection; Bold indicates significant P values, NA: not available; MST: median survival time; HR: hazard ratio; $95 \%$ CI: $95 \%$ confidence interval. 
Table 3. Joint-effect analysis of DUXAP8 and RNF2 for overall survival

\begin{tabular}{|c|c|c|c|c|c|c|c|c|}
\hline \multirow[t]{2}{*}{ Group } & \multirow[t]{2}{*}{ DUXAP8 expression } & \multirow[t]{2}{*}{ RNF2 } & \multicolumn{6}{|c|}{ Overall survival } \\
\hline & & & Events/total & MST (Days) & Crude HR $(95 \% \mathrm{CI})$ & Crude $P$ value & Adjusted HR (95\%CI) & Adjusted $P$ value ${ }^{\phi}$ \\
\hline $\bar{A}$ & Low & Low & $39 / 120$ & 2116 & Ref. & $<0.0001$ & Ref. & 0.001 \\
\hline \multirow[t]{2}{*}{ B } & Low & High & $35 / 130$ & 2542 & $1.001(0.633-1.584)$ & 0.995 & $1.030(0.626-1.694)$ & 0.908 \\
\hline & High & Low & & & & & & \\
\hline $\mathrm{C}$ & High & High & $56 / 120$ & 837 & $2.268(1.498-3.433)$ & $<0.001$ & 2.184 (1.364-3.497) & 0.001 \\
\hline
\end{tabular}

Note: $\phi$ : P values were adjusted for radical resection, tumor stage and HBV infection; Bold indicates significant P values, NA: not available; MST: median survival time; HR: hazard ratio; $95 \%$ CI: $95 \%$ confidence interval.

Table 4. Construction of risk score model

\begin{tabular}{llllll}
\hline Variables & $\beta$ & SE & Wald & $P$ value & HR (95\% CI) \\
\hline Tumor stage I & & & 11.611 & 0.003 & 0.567 \\
Stage II & 0.153 & 0.268 & 0.328 & 0.001 & $1.166(0.690-1.970)$ \\
Stage III + IV & 0.734 & 0.224 & 10.683 & 0.848 & $2.083(1.341-3.233)$ \\
Radical resection & 0.068 & 0.356 & 0.037 & 0.001 & $1.071(0.533-2.152)$ \\
HBV infection & -0.896 & 0.264 & 11.545 & 0.064 & $0.408(0.244-0.685)$ \\
DUAXP8 & 0.382 & 0.206 & 3.432 & 0.037 & $1.465(0.978-2.195)$ \\
RNF2 & 0.433 & 0.207 & 4.366 & & $1.542(1.027-2.315)$ \\
\hline
\end{tabular}

Note: SE: standard error, HR: hazard ratio, CI: confidence interval.

Table 5. Overall survival analysis of risk score model

\begin{tabular}{llllll}
\hline Group & \multicolumn{2}{l}{ Overall survival } & & & \\
\cline { 2 - 6 } & Events/total & MST (Days) & Crude HR (95\%CI) & Crude $P$ value & Adjusted HR (95\%CI) \\
\hline Risk score model & $54 / 185$ & 2131 & Ref. & $\mathbf{0 . 0 0 1}$ & Ref. \\
Low risk & $76 / 185$ & 1271 & $\mathbf{1 . 8 0 8 ~ ( 1 . 2 7 4 - 2 . 5 6 5 )}$ & & $\mathbf{0 . 0 1 0}$ \\
High risk & & & $\mathbf{1 . 6 7 2}(\mathbf{1 . 1 3 0 - 2 . 4 7 7 )}$ \\
\hline
\end{tabular}

Note: $\phi$ : P values were adjusted for radical resection, tumor stage and HBV infection; Bold indicates significant $\mathrm{P}$ values.

Abbreviations: NA: not available; MST: median survival time; HR: hazard ratio; $95 \% \mathrm{CI}$ : 95\% confidence interval.

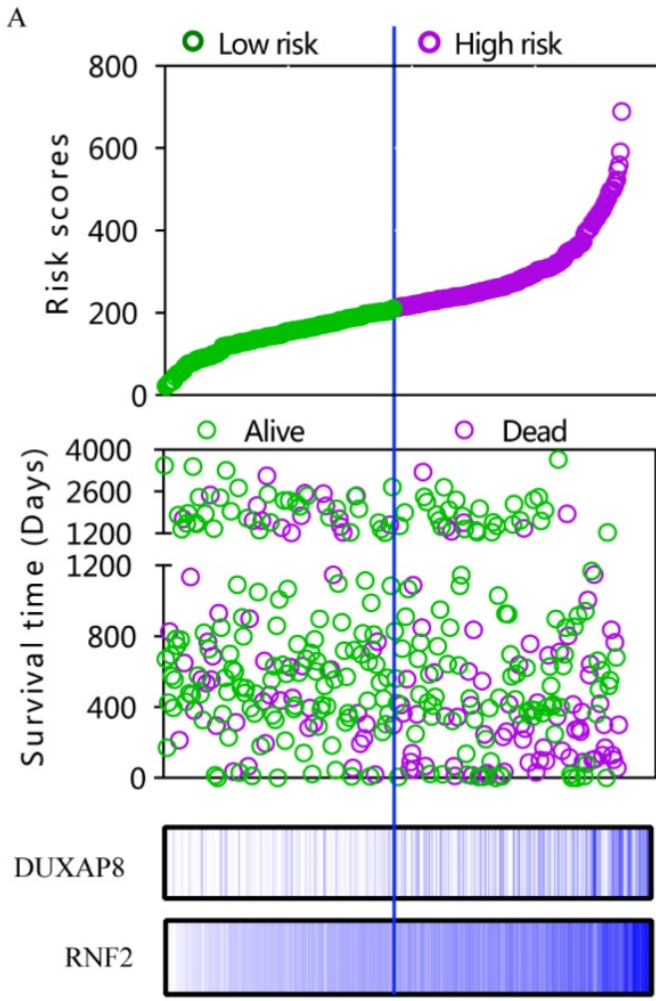

B

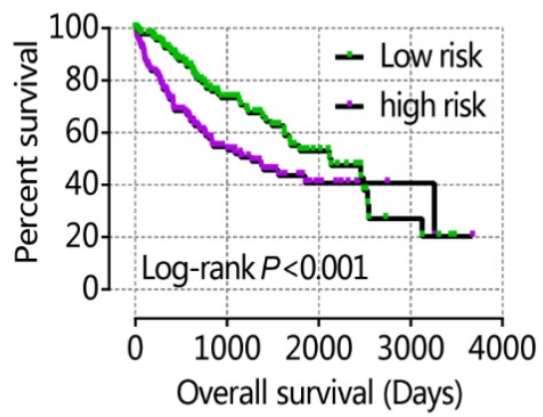

$\mathrm{C}$

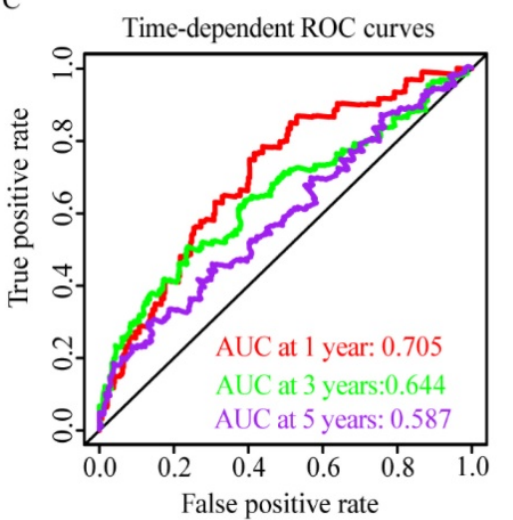

Figure 6. Risk score model, Kaplan-Meier plot, and time-dependent ROC curves. A: risk score model constructed using risk scores, patient survival status, DUXAP8 and RNF2 expression heat maps; B: Kaplan-Meier plot of low and high risk groups; C: Time-dependent ROC curves of 1, 3, and 5year OS. 
A

Points

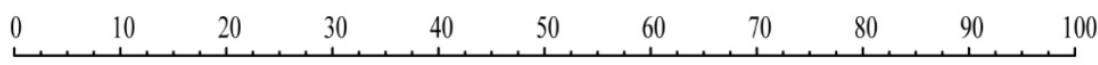

Tumor stage

Radical resection

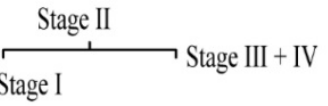

HBV infection

Yes $\quad$ No

HBV infection

DUXAP8

No

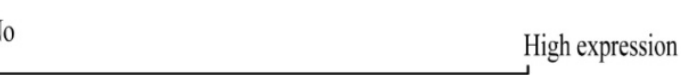

RNF2

Low expression

High expression

Total Points

Low expression

1-year OS

3-year OS

5-year OS

\begin{tabular}{lllllllllllll}
\hline 0 & 20 & 40 & 60 & 80 & 100 & 120 & 140 & 160 & 180 & 200 & 220 & 240
\end{tabular}

B

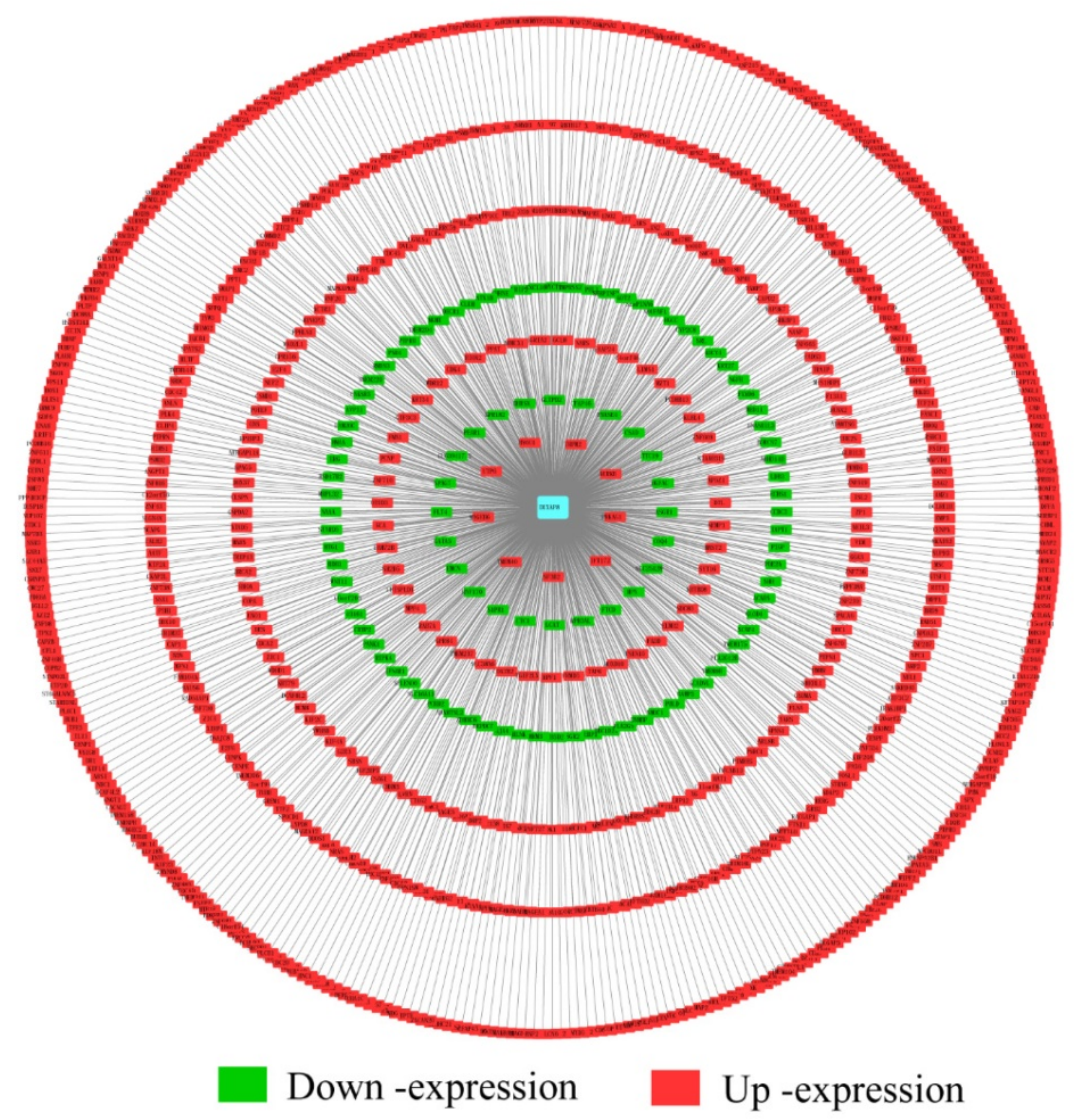

Figure 7. Nomogram and co-expression network of DUXAP8 and co-expression-related protein-coding genes. A: Nomogram constructed using DUXAP8, RNF2, tumor stage, radical resection, and hepatitis B virus infection status; B: Co-expression network between DUXAP8 and the co-expression-related protein-coding genes.

\section{Assessment of molecular mechanism using GSEA}

GSEA analysis of GO terms for DUXAP8 revealed that it was enriched in mitotic nuclear division, cell cycle phase transition, positive regulation of cell cycle phase transition, meiotic cell cycle, cell division, centrosome, chromosome condensation, and histone binding (Figure 8A-I). KEGG pathway analysis revealed that DUXAP8 was associated with fatty acid metabolism, tryptophan metabolism, and the citrate cycle (TCA cycle) pathways (Figure 8J-L). For RNF2, GO analysis 
revealed that it enriched in cell division, mitotic nuclear division, cell cycle checkpoint, sister chromatid segregation, chromatin modification and histone binding (Figure 9A-I). KEGG pathways analysis showed that RNF2 was associated with oxidative phosphorylation, PPAR signaling pathway, and the TCA cycle pathways (Figure 9J-L).

\section{Construction of co-expression matrix, GGI and PPI network}

The co-expression matrix of DUXAP8 and PCGs revealed that most of them were positively correlated with each other. In the matrix, deep blue color indicated highly and positively correlated interactions (Figure 10A). The GGI network indicated that CNTNAP4, MKRN3, DGKI, GABRA3, MAGEA6, and $M A G E A 1$ were co-expressed with other genes (Figure 10B). Many $M A G E$ family members interacted with them. Proteins encoded by CNTNAP4, GABRA3, DGKI, MAGEA6, MAGEA3 and MAGEA1 genes show co-expressional and co-occurrence relationship (Figure 10C).
A

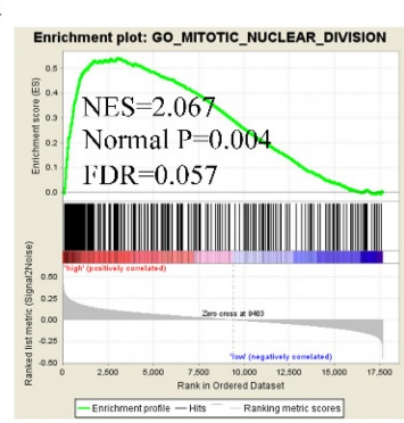

D

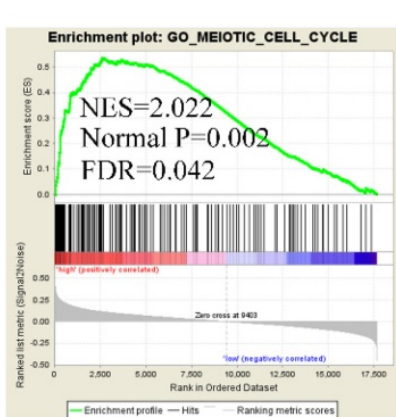

G

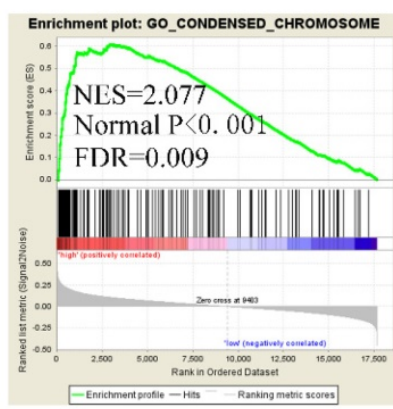

$\mathrm{J}$

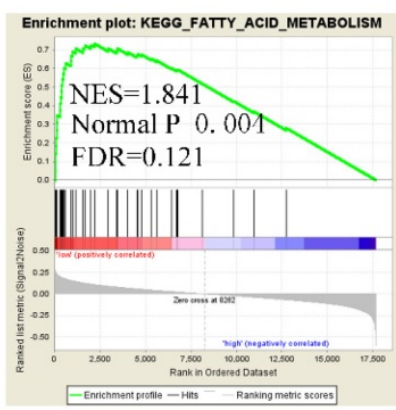

B

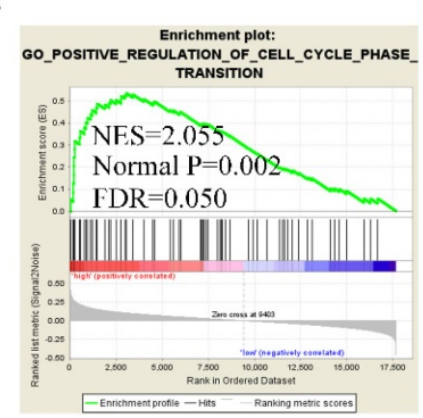

E

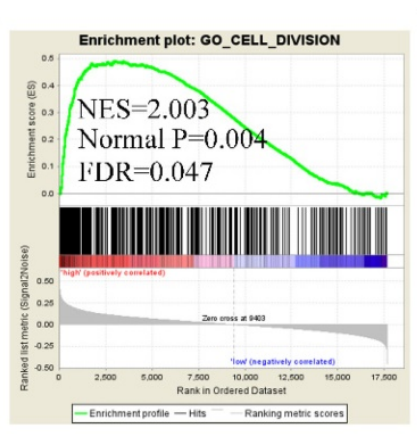

$\mathrm{H}$

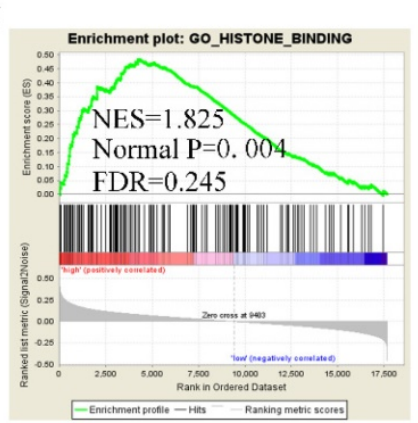

$\mathrm{K}$

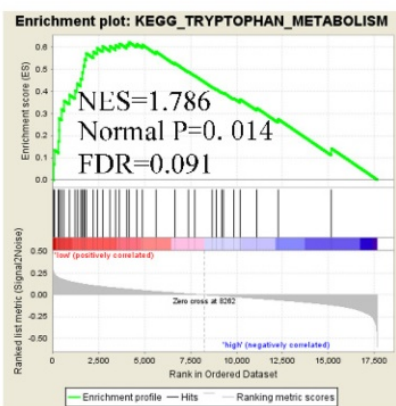

$\mathrm{C}$

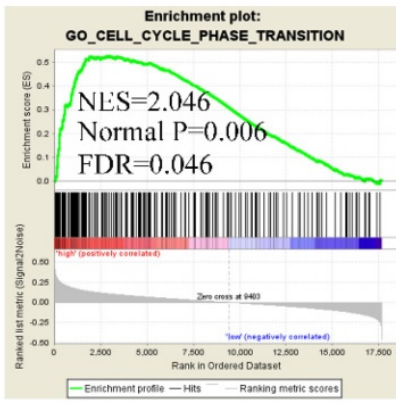

$\mathrm{F}$

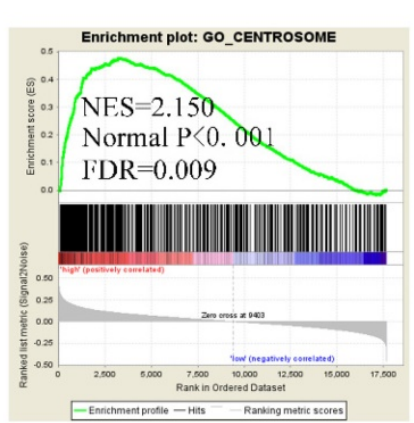

I

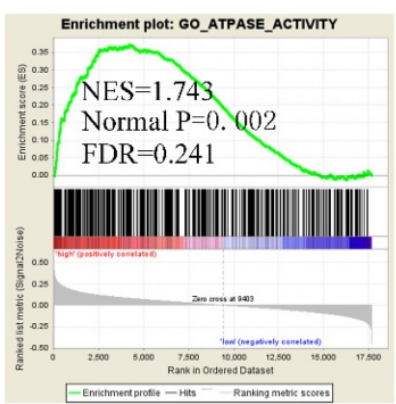

L

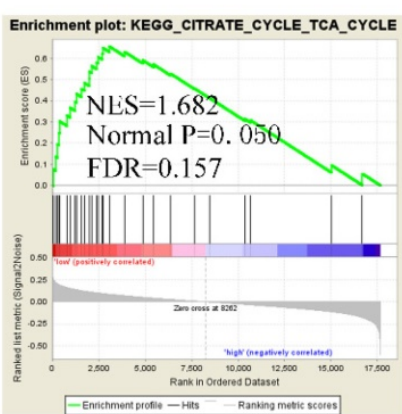

Figure 8. Gene set enrichment analysis of DUXAP8 of gene ontologies and KEGG pathways. A-I: Gene ontology results of DUXAP8; J-L: KEGG pathway results of DUXAP8. 
A

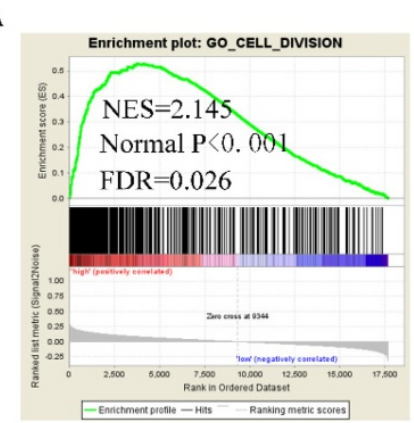

D

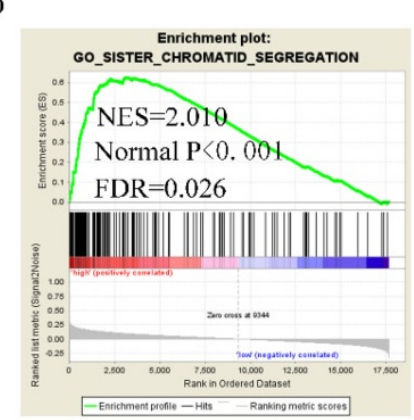

G

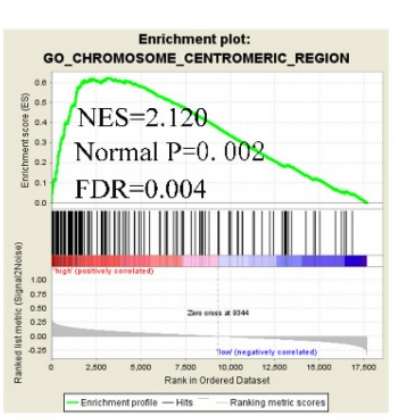

J

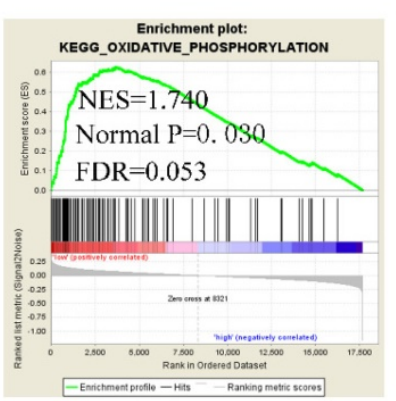

B
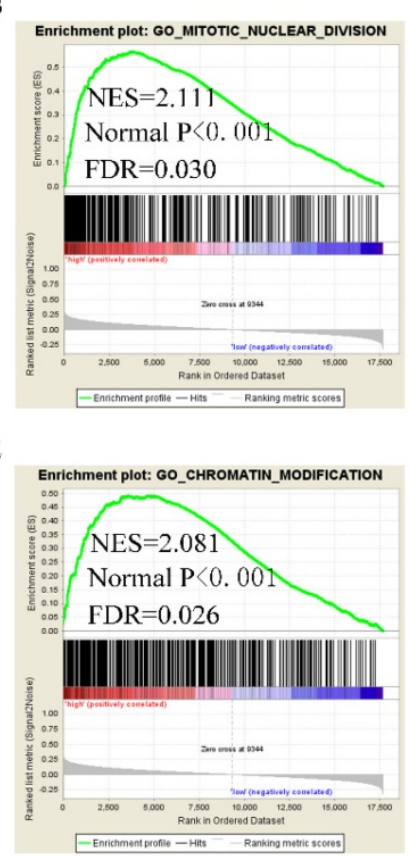

$\mathrm{H}$

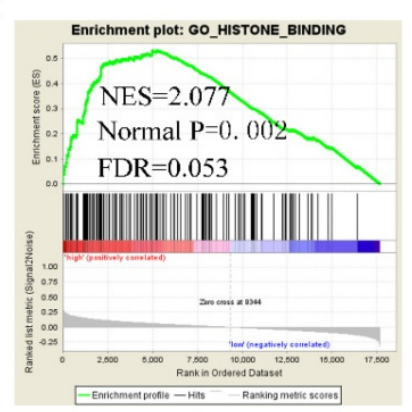

K

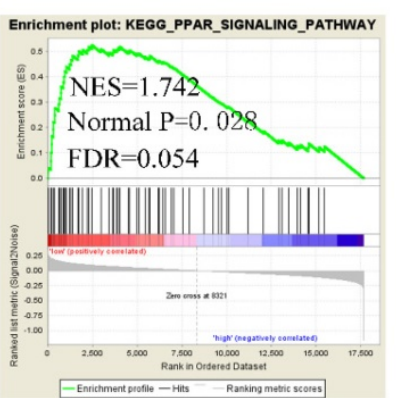

C

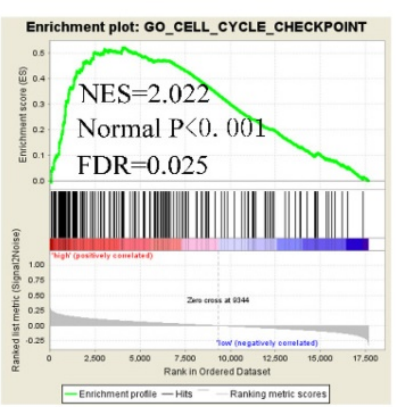

$\mathrm{F}$

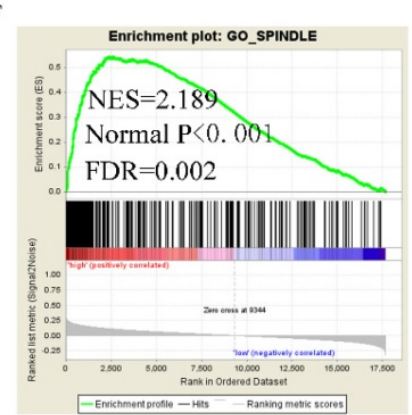

I

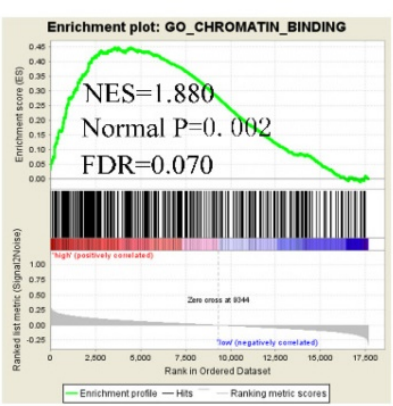

L

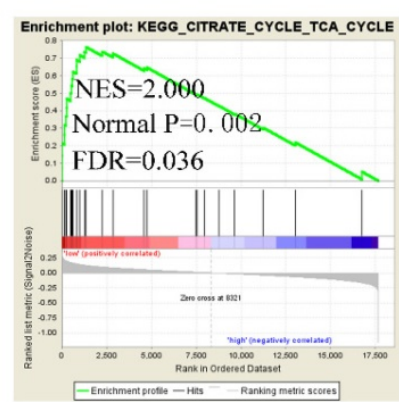

Figure 9. Gene set enrichment analysis of RNF2 of gene ontologies and KEGG pathways. A-l: Gene ontology results of RNF2; J-L: KEGG pathway results of RNF2.

\section{Identification of pharmacological targets}

DEGs were identified using edgeR and presented in heatmap and volcano plots as shown in Figure 11. Potential drugs targeting the DEGs were: cinchonine, bumetanide and amiprilose (Table 6). Detailed drug information is provided in Supplementary Table 3 . The 2D and 3D structures of these three chemical compounds are in Figure 12. GO analysis was performed for the DEGs and results are shown in Figure 13. The DEGs were enriched in the synapse, extracellular region, postsynaptic membrane, ion channel complex, multicellular organismal development, and extracellular ligand-gated ion channel activity. The top $10 \mathrm{GO}$ terms and KEGG pathways were: chemical synaptic transmission, ligand-gated ion channel activity, extracellular region, postsynaptic membrane, extracellular ligand-gated ion channel activity, neuroactive ligand-receptor interaction, and retrograde endocannabinoid signaling (Table 7). Detailed results of GO terms and KEGG pathways enrichment are shown in Supplementary Tables 4 and 5 , respectively. 


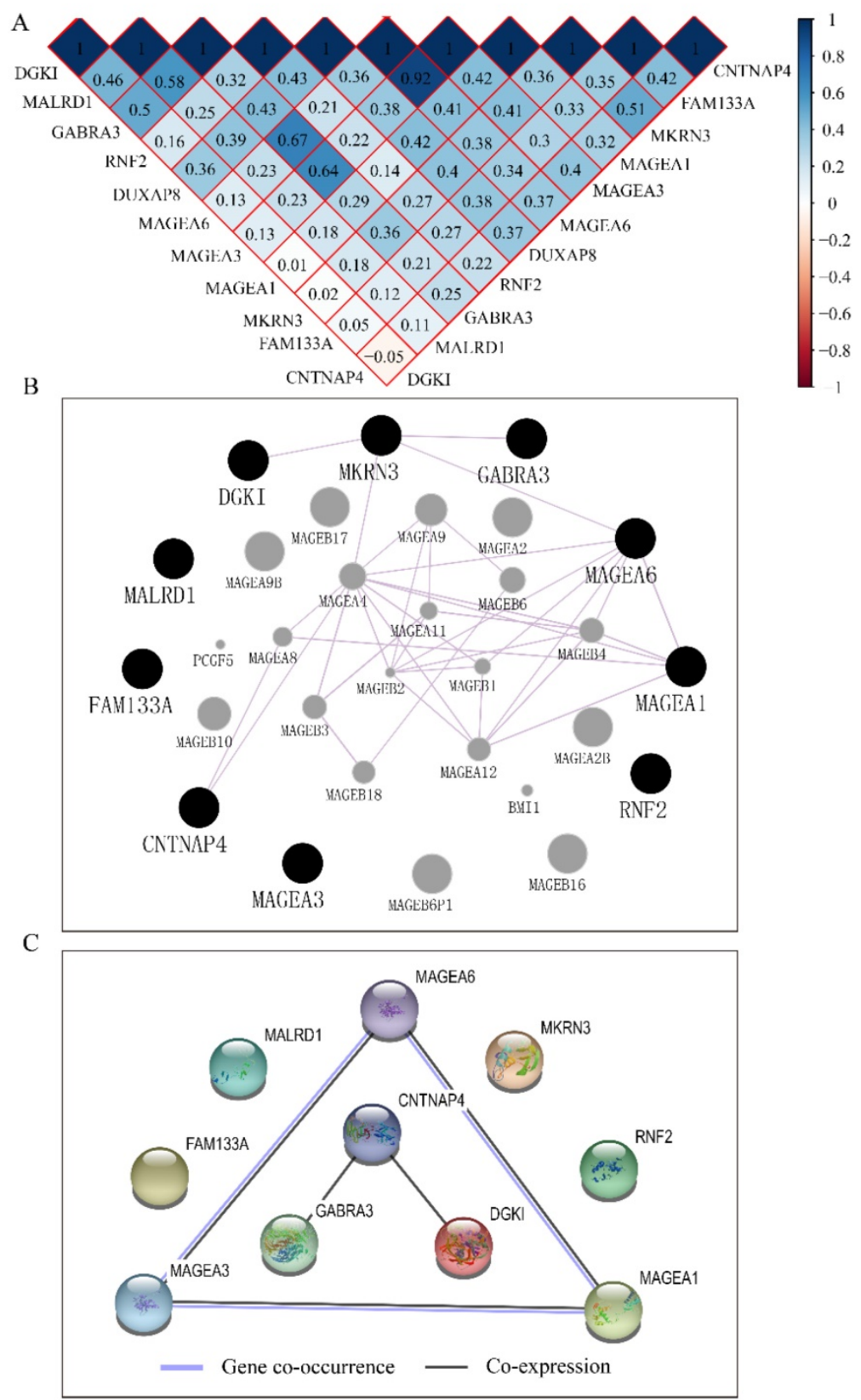

Figure 10. Co-expression matrix and gene-gene interaction and protein-protein interaction networks of LINC00668 and co-expression-related protein-coding genes. A: Co-expression matrix among DUXAP8, RNF2, MAGEAI, GABRA3, MKRN3, FAMI33A, MAGEA3, CNTNAP4, MAGEA6, MALRDI, and DGKI; B: Co-expression network of gene-gene interactions among protein-coding genes; C: Protein-protein interaction network among RNF2, MAGEAI, GABRA3, MKRN3, FAMI33A, MAGEA3, CNTNAP4, MAGEA6, MALRDI, and DGKI.

Table 6. Top 7 pharmacological target and drugs of DUXAP8

\begin{tabular}{lllll}
\hline Name & PubChem CID & Mean & Enrichment & P value \\
\hline D-Cinchonine & 90454 & -0.411 & -0.755 & 0.00734 \\
Bumetanide & 2471 & -0.407 & -0.708 & 0.01486 \\
Scopolamine & 300322 & 0.383 & 0.694 & 0.01842 \\
Trolox C & 40634 & 0.164 & 0.690 & 0.01920 \\
Harmaline & 3564 & 0.430 & 0.660 & 0.03083 \\
Cefotetan & 53025 & 0.504 & 0.736 & 0.03609 \\
Amiprilose & 9798098 & -0.413 & -0.641 & 0.03981 \\
\hline
\end{tabular}

\section{Validations of potential significance of PCGs in public databases}

Then, we further validated the potential significance of PCGs in HCC using HCCDB and oncomine databases. The diagnostic significance of MAGEA1, MKRN3, and DGKI was validated using oncomine database, which suggested diagnostic significance of MAGEA1 (AUC $=0.737, \quad \mathrm{P}=0.024$, Supplementary Figure 1A, D) and DGKI (AUC $=0.726$, $\mathrm{P}=0.031$, Supplementary Figure 1C, F), but not $M K R N$ (AUC $=0.655, \mathrm{P}=0.058$, Supplementary Figure $1 \mathrm{~B}, \mathrm{E}$ ). The prognostic significance of RNF2 was consistently validated in $\mathrm{HCCDB}$, demonstrating the prognostic values of it in HCC (Log-rank P=0.001, 0.044, respectively, Supplementary Figure 1G-H). 
Table 7. Top 10 gene ontologies and KEGG pathways of differentially expressed genes

\begin{tabular}{|c|c|c|c|c|}
\hline Category & Term & Count & $\%$ & Pvalue \\
\hline $\mathrm{BP}$ & GO: 0007268 chemical synaptic transmission & 19 & 4.241071429 & $3.86 \mathrm{E}-06$ \\
\hline MF & GO: 0015276 ligand-gated ion channel activity & 8 & 1.785714286 & $6.26 \mathrm{E}-06$ \\
\hline $\mathrm{CC}$ & GO: 0005576 extracellular region & 62 & 13.83928571 & $9.28 \mathrm{E}-06$ \\
\hline $\mathrm{BP}$ & GO: 0035094 response to nicotine & 8 & 1.785714286 & $1.08 \mathrm{E}-05$ \\
\hline $\mathrm{CC}$ & GO: 0045211 postsynaptic membrane & 17 & 3.794642857 & $1.40 \mathrm{E}-05$ \\
\hline $\mathrm{CC}$ & GO: 0005615 extracellular space & 53 & 11.83035714 & 2.78E-05 \\
\hline MF & GO: 0005230 extracellular ligand-gated ion channel activity & 7 & 1.5625 & $4.40 \mathrm{E}-05$ \\
\hline $\mathrm{BP}$ & GO: 0007586 digestion & 9 & 2.008928571 & $5.29 \mathrm{E}-05$ \\
\hline MF & GO: 0005179 hormone activity & 10 & 2.232142857 & 1.13E-04 \\
\hline $\mathrm{BP}$ & GO: 0007271 synaptic transmission, cholinergic & 7 & 1.5625 & $1.16 \mathrm{E}-04$ \\
\hline KEGG pathway & hsa04080: Neuroactive ligand-receptor interaction & 30 & 6.696428571 & 1.09E-14 \\
\hline KEGG pathway & hsa04972: Pancreatic secretion & 10 & 2.232142857 & 5.19E-05 \\
\hline KEGG pathway & hsa04723: Retrograde endocannabinoid signaling & 9 & 2.008928571 & $5.48 \mathrm{E}-04$ \\
\hline KEGG pathway & hsa05033: Nicotine addiction & 6 & 1.339285714 & 8.05E-04 \\
\hline KEGG pathway & hsa04727: GABAergic synapse & 8 & 1.785714286 & $9.70 \mathrm{E}-04$ \\
\hline KEGG pathway & hsa04974: Protein digestion and absorption & 8 & 1.785714286 & 0.001191854 \\
\hline KEGG pathway & hsa05032: Morphine addiction & 7 & 1.5625 & 0.006772348 \\
\hline KEGG pathway & hsa05218: Melanoma & 5 & 1.116071429 & 0.042731956 \\
\hline KEGG pathway & hsa04725: Cholinergic synapse & 6 & 1.339285714 & 0.055498778 \\
\hline KEGG pathway & hsa00053: Ascorbate and aldarate metabolism & 3 & 0.669642857 & 0.089180199 \\
\hline
\end{tabular}

Note: KEGG, Kyoto Encyclopedia of Genes and Genomes, BP: biological process, CC: cellular component, MF: molecular function, FDR: false discovery rate.

A
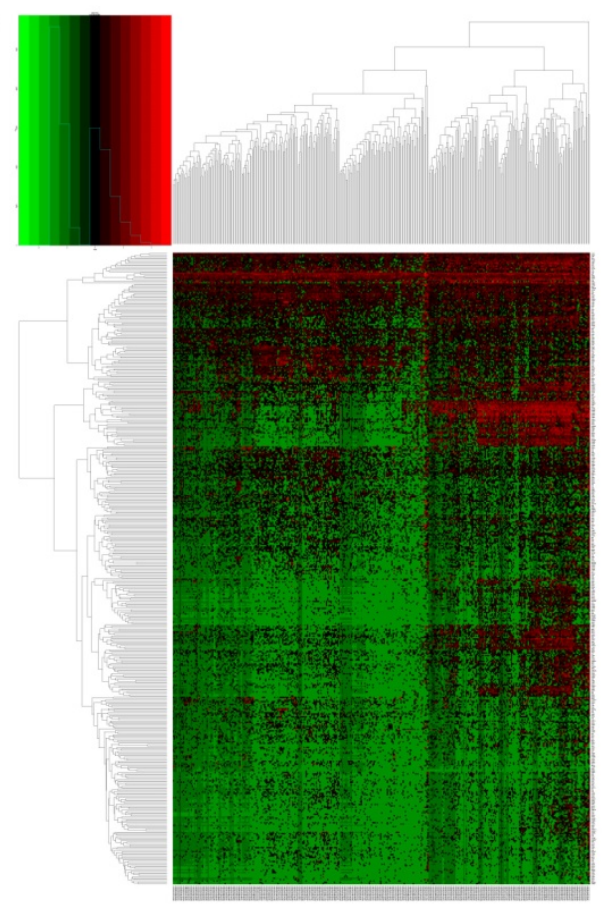

B

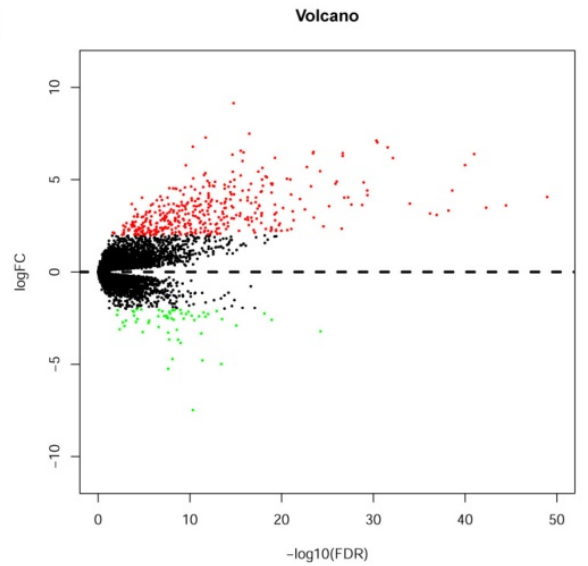

Figure 11. Heatmap and volcano plot of differentially expressed genes of DUXAP8. A: Heatmap of differentially expressed genes of DUXAP8; B: Volcano plot of differentially expressed genes of DUXAP8. 
$\Lambda$

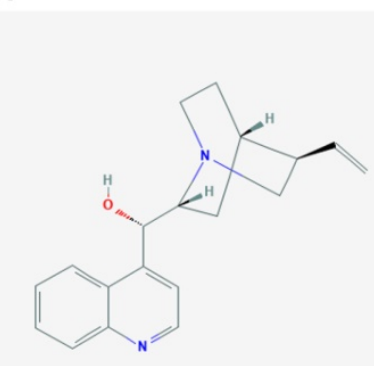

$\mathrm{D}$

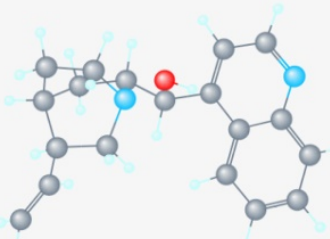

B

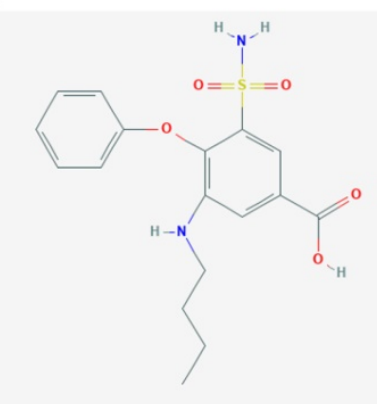

E

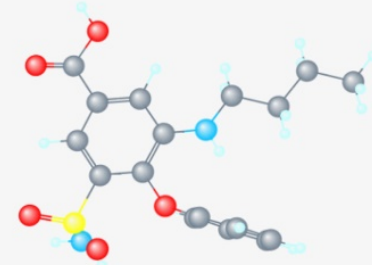

C

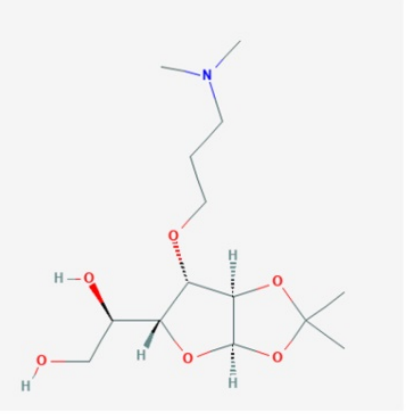

F

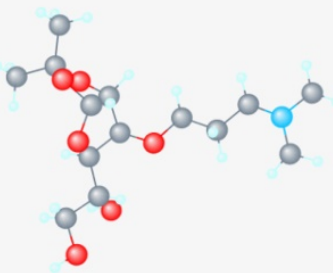

Figure 12. 2D and 3D structure of the chemical compound of the 3 target drugs. A-C: $2 D$ structure of cinchonine, bumetanide and amiprilose, respectively; D-F: 3D structure of cinchonine, bumetanide and amiprilose, respectively.

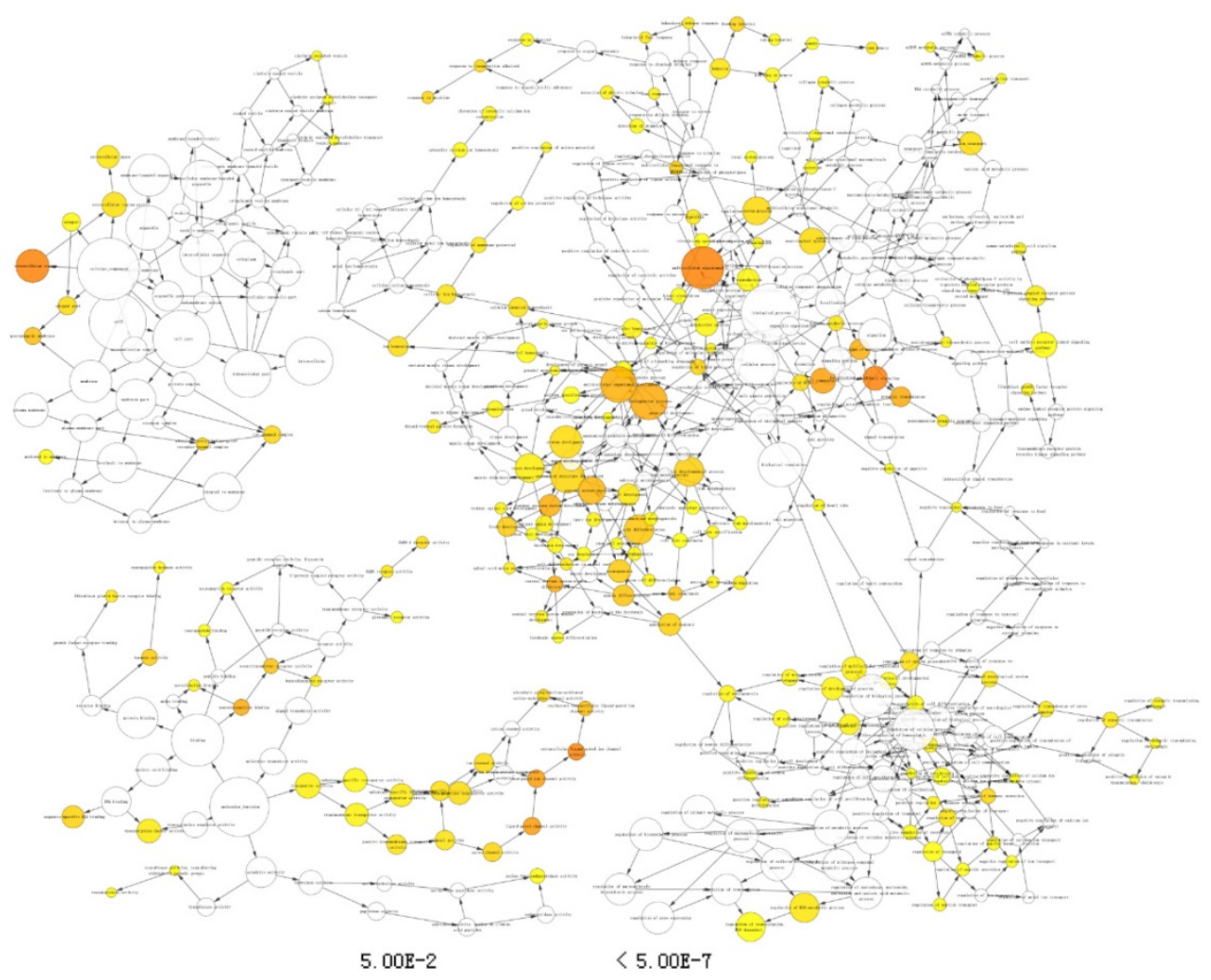

Figure 13. Enriched gene ontology terms network using differentially expressed genes.

\section{Discussion}

The present study investigated the prognostic value of DUXAP8 and its PCGs. We found that
DUXAP8, RNF2, MAGEA1, GABRA3, MKRN3, FAM133A, MAGEA3, CNTNAP4, MAGEA6, and DGKI are differentially expressed in liver tissues and are overexpressed in tumor tissues. Diagnostic analysis 
indicated that DUXAP8, MEGEA1, MKRN3, and DGKI are potential diagnostic biomarkers of HCC while DUXAP8 and RNF2 are potential prognostic biomarkers of HCC. The joint-effect analysis of DUXAP8 and the PCGs showed better diagnostic and prognostic value compared to individual application. The constructed risk score model and nomogram showed good prediction for HCC prognosis. Molecular mechanism analyses revealed that DUXAP8 and RNF2 are involved in mitotic nuclear division, cell division, regulation of cell cycle, phase transition, histone binding, oxidative phosphorylation, PPAR signaling pathway, and the TCA cycle. In addition, three target drugs; cinchonine, bumetanide, and amiprilose, were identified as targets of DUXAP8 using the Connectivity Map database. They are therefore likely to be ideal treatments for HCC treatment. Then, diagnostic and prognostic significance of PCGs were validated in HCCDB and oncomine databases.

Accumulating evidence indicates that non-coding RNAs regulate several physiological and pathological biological processes $(40,41)$. LncRNAs have been proposed to be potential diagnostic markers and therapeutic agents for various diseases (42). Dysregulation of lncRNAs may alter development of tumors (42). For instance, it is emerging that lncRNAs participate in tumorigenesis more actively than has been previously reported (42). Differential expression patterns of lncRNAs affect cell transformation, tumorigenesis, and metastasis (43). For instance, H19, HOTAIR, MALAT1, TUG1, GAS5, and CCAT1, were found to play important roles in tumor initiation and development (44-49). Numerous cancer-related lncRNAs modulates tumor invasion and metastasis processes (50-52). The transforming growth factor- $\beta$ promotes the expression of ATB lncRNA in HCC cell lines, hence enhancing mesenchymal cell transition, cell invasion, and organ colonization of HCC cell lines (53).

DUXAP8, a 2107 bp RNA, was initially found to be overexpressed in gastric cancer (GC) tissues, its overexpression resulted in larger tumor size, advanced tumor stage, lymphatic metastasis, and poor prognosis of GC patients (54). It has been reported that DUXAP8 enhances GC cell proliferation and tumorigenesis, partly by epigenetically silencing PLEKHO1 expression through binding to PRC2, making it a potential biomarker for GC diagnosis and therapy (54). Hongzhi et al. found that DUXAP8 was overexpressed in pancreatic cancer (PC) tissues indicating poor OS in PC patients making it a potential therapeutic target (18). They also reported that DUXAP8 overexpression resulted in larger tumor size, advanced pathologic stage and poor OS of PC.
This accelerates cell proliferation and tumorigenesis, partly by epigenetically silencing transcription of CDKN1A and KLF2, and by binding to EZH2 and LSD1 (18). Comprehensive profiling analysis revealed that DUXAP8 is upregulated in Bladder Cancer (BC). Its downregulation decreases cell growth, colony formation, invasion capacity and induces apoptosis of BC cells (20). Enrichment analysis of SNHG12 and DUXAP8 co-expressed PCGs found that they are involved in the cell cycle, focal adhesion, and PI3K-AKT signaling pathways (20). They also reported that DUXAP8 may regulate tumorigenesis and progression of $B C(20)$.

Zheng et al. found that DUXAP8 is upregulated in renal cell carcinoma (RCC) tissues and promotes the proliferation and invasion of RCC cells by downregulating microRNA-126 expression (19). They also reported that DUXAP8 positively regulated RCC tumorigenesis and development (19). Elsewhere, DUXAP8 was found to be highly expressed in esophageal squamous cell cancer (ESCC) tissues and was associated with tumor stage, lymph node metastasis, and correlated with poor survival of ESCC patients (21). Functional experiments suggest that DUXAP8 modulates the occurrence of ESCC via the Wnt- $\beta$-catenin pathway. Specifically, it promotes cell proliferation, colony formation, and invasion of ESCC cells (21).

A genome-wide analysis revealed that DUXAP8 was highly expressed in esophageal cancer. GO enrichment analysis for DUXAP8 and its co-expressing PCGs showed that they were enriched in the cell cycle, cell division and DNA repair, suggesting an important role in the tumorigenesis and progression of esophageal cancer (24). It has been reported that knockdown of DUXAP8 resulted in clear cell cycle arrest in the G0/G1 phase ini non-small lung cancer cell lines, H1299, and H1975, which further decreased cyclin D1, CDK2, CDK4 and CDK6 expression in cell cycle process (23). Similarly, DUXAP8 was up-regulated in pancreatic cancer tissues. Knockdown of DUXAP8 expression arrested the cell cycle at the G0/G1 phase and induced apoptosis of pancreatic cancer cell lines (18). DUXAP8, SNHG12, and their PCGs were enriched in the cell cycle, focal adhesion, and PI3K-Akt signaling pathway (20). However, Hong-wei Ma's study using cell apoptosis and cell cycle regulation as factors reflecting cell growth of gastric cancer, revealed that the proportion of apoptotic cells were significantly decreased but not in the proportion in different phases (54).

We found cinchonine, bumetanide, and amiprilose as the potential drugs targeting DUXAP8 in HCC. Cinchonine $\left(\mathrm{C}_{19} \mathrm{H}_{22} \mathrm{~N}_{2} \mathrm{O}\right)$ is a natural 
compound used as an antimalarial drug (55). It exerts antitumor effects with high activity and low toxicity (56). Moreover, cinchonine inhibited cell proliferation and promoted apoptosis by activating caspase-3 dependence in human liver cancer cells (57). Similarly, it was reported that cinchonine induced apoptosis of Hela and A549 cells by targeting TRAF6, suggesting that cinchonine has antitumor effects (58). Bumetanide is a commonly used diuretic drug in clinical practice. A previous study showed that bumetanide, a SLC12A1 antagonist, inhibited cell proliferation, tumorigenesis, and metastasis in HCC cell lines (59). The study also suggested that bumetanide slows down tumor growth by interfering with the cell cycle rather than by inducing cytotoxicity (59). Studies testing the ability of bumetanide to enhance tumor necrosis in a rat model of N1-S1 HCC. They found that bumetanide treatment increased tumor necrosis in N1-S1 HCC during transarterial embolization compared to during transarterial embolization alone (60). Amiprilose is a synthetic carbohydrate used for patients with rheumatoid arthritis. It has anti-inflammatory and immunomodulatory properties (61). None has reported whether it has any effects on liver cancer. Our findings showed that cinchonine, bumetanide, and amiprilose are candidate drugs that target DUXAP8 in HCC. However, the specific mechanisms of action of these drugs in HCC deserves further studies.

Our study indicates that higher expression of DUXAP8 correlated with poor prognosis in HCC. We also found that DUXAP8 is involved in the mitotic nuclear division, cell division, regulation of cell cycle phase transition, histone binding, oxidative phosphorylation, and the TCA cycle pathways. Therefore, we infer that DUXAP8 functions as an oncogene in HCC. This conclusion is in agreement with previous reports in ESCC, PC, BC, GC, and RCC.

Live cell imaging or readouts of active cellular processes have revealed that lncRNAs play various roles in cellular pathologies and can influence the efficacy of new therapeutic targets for cancer (62). Our study identified three potential drugs targeting DUXAP8 in HCC. Although several related studies focusing on DUXAP8 in HCC were previously reported (63-65), our study has several main findings that could distinguished from them, such as in both diagnostic and prognostic significance of DUXAP8 and it-related PCGs, construction of risk score model and nomogram, potential target drugs toward DXUAP8 aspects.

We show that SNF2 is overexpressed in tumor tissues leading to poor patient survival. Molecular mechanism results demonstrate that SNF2 is involved in cell division, mitotic nuclear division, cell cycle checkpoint, sister chromatid segregation, chromatin modification, histone binding, oxidative phosphorylation, PPAR signaling pathway, and the TCA cycle. Therefore, we speculate that SNF2 is an oncogene in HCC A recent study showed that downregulation of SNF2, a co-expression-related gene of DUXAP8, decreases cell growth and metastases of HCC cells. (66). The study showed that RNF2 promoted HCC cell proliferation by accelerating cell cycle progression (66). Similar findings have been reported for SNF2 in several malignancies(67). In conclusion, our study is consistent with previous studies showing that SNF2 is an oncogene in HCC.

This study for the first time demonstrates the oncogenic role, diagnostic and prognostic value of DUXAP8 in HCC. This study has some limitations that need to be recognized. First, our findings concerning DUXAP8 and its PCGs need to be validated using a larger population. Second, well-designed functional trials are necessary to identify deeper mechanisms of DUXAP8 and its PCG in HCC. Thus, further clinical trials are required to assess the translational potential of DUXAP8.

\section{Conclusions}

This study investigated the functions of DUXAP8 and its PCGs in HCC. We found that DUXAP8 and its PCGs are differentially expressed in the liver and overexpressed in tumor tissues. Diagnostic and prognostic analysis indicated that DUXAP8, MEGEA1, MKRN3, and DGKI can be used to diagnosis HCC, while DUXAP8 and RNF2 can be used to predict the prognosis of HCC. In addition, we found that DUXAP8 and its PCGs demonstrated better diagnostic and prognostic performance when these factors were used jointly as opposed to single application. A risk score model and nomogram exhibited good prognostic prediction performance of DUXAP8 and its PCGs in HCC. Molecular analyses revealed that DUXAP8 and PCGs are involved in mitotic nuclear division, cell division, regulation of cell cycle phase transition, oxidative phosphorylation, and PPAR signaling pathway. Furthermore, three potential target drugs; cinchonine, bumetanide, and amiprilose, which were identified as candidate drugs targeting DUXAP8 in HCC. This study for the first time demonstrates the oncogenic role, diagnostic and prognostic value of DUXAP8 in HCC. Thus, further clinical trials are required to assess the translational potential of DUXAP8.

\section{Supplementary Material}

Supplementary figure.

http://www.jcancer.org/v11p6140s1.pdf 
Supplementary tables.

http://www.jcancer.org/v11p6140s2.xlsx

\section{Acknowledgements}

This work was supported in part by the National Natural Science Foundation of China (No.: 81560535, 81802874, 81072321, 30760243, 30460143 and 30560133), Natural Science Foundation of Guangxi Province of China (Grant No. 2018GXNSFBA138013, 2018GXNSFAA050119), 2009 Program for New Century Excellent Talents in University (NCET), Guangxi Natural Sciences Foundation (No.: GuiKeGong 1104003A-7), and Guangxi Health Ministry Medicine Grant (Key-Scientific Research-Grant Z201018). The present study is also partly supported by Scientific Research Fund of the Health and Family Planning Commission of Guangxi Zhuang Autonomous Region (Z2016318), Key laboratory of High-Incidence-Tumor Prevention \& Treatment (Guangxi Medical University), Ministry of Education (GKE2018-01, GKE2019-11, GKE-ZZ202009), The Basic Ability Improvement Project for Middle-aged and Young Teachers in Colleges and Universities in Guangxi (2018KY0110). As well as, the present study is also partly supported by Research Institute of Innovative Think-tank in Guangxi Medical University (The gene-environment interaction in hepatocarcinogenesis in Guangxi HCCs and its translational applications in the HCC prevention). We would also acknowledge the supported by the Key laboratory of High-Incidence-Tumor Prevention \& Treatment (Guangxi Medical University), Ministry of Education. The authors would like to acknowledge those researchers for their contribution on open access of Connectivity Map and GEPIA website. In addition, the authors would like to acknowledge invaluable help from peer reviewers.

\section{Availability of data and materials}

The datasets analyzed during the current study are available from the corresponding author on reasonable request.

\section{Competing Interests}

The authors have declared that no competing interest exists.

\section{References}

1. Bray F, Ferlay J, Soerjomataram I, Siegel RL, Torre LA, Jemal A. Global cancer statistics 2018: GLOBOCAN estimates of incidence and mortality worldwide for 36 cancers in 185 countries. CA Cancer J Clin. 2018;68(6):394-424.

2. Yang JD, Roberts LR. Epidemiology and management of hepatocellular carcinoma. Infectious disease clinics of North America. 2010;24(4):899-919, viii.

3. Testino G, Leone S, Patussi V, Scafato E, Borro P. Hepatocellular carcinoma: diagnosis and proposal of treatment. Minerva Med. 2016;107(6):413-26.
4. Gomaa AI, Khan SA, Toledano MB, Waked I, Taylor-Robinson SD. Hepatocellular carcinoma: epidemiology, risk factors and pathogenesis. World J Gastroenterol. 2008;14(27):4300-8.

5. Varnholt H, Drebber U, Schulze F, Wedemeyer I, Schirmacher P, Dienes HP, et al. MicroRNA gene expression profile of hepatitis $C$ virus-associated hepatocellular carcinoma. Hepatology. 2008;47(4):1223-32.

6. Farazi PA, DePinho RA. Hepatocellular carcinoma pathogenesis: from genes to environment. Nature reviews Cancer. 2006;6(9):674-87.

7. Huang C, Zhuang W, Feng H, Guo H, Tang Y, Chen H, et al. Analysis of therapeutic effectiveness and prognostic factor on argon-helium cryoablation combined with transcatheter arterial chemoembolization for the treatment of advanced hepatocellular carcinoma. J Cancer Res Ther. 2016;12(Supplement):C148-c52.

8. Wakayama K, Kamiyama T, Yokoo H, Orimo T, Shimada S, Einama T, et al. Huge hepatocellular carcinoma greater than $10 \mathrm{~cm}$ in diameter worsens prognosis by causing distant recurrence after curative resection. Journal of surgical oncology. 2017;115(3):324-9.

9. Forner A, Reig M, Bruix J. Hepatocellular carcinoma. Lancet. 2018;391(10127):1301-14.

10. Tien AJ, Chien CY, Chen YH, Lin LC, Chien CT. Fruiting Bodies of Antrodia cinnamomea and Its Active Triterpenoid, Antcin $\mathrm{K}$, Ameliorates N-Nitrosodiethylamine-Induced Hepatic Inflammation, Fibrosis and Carcinogenesis in Rats. The American journal of Chinese medicine. 2017;45(1):173-98.

11. He X, Sun F, Guo F, Wang K, Gao Y, Feng Y, et al. Knockdown of Long Noncoding RNA FTX Inhibits Proliferation, Migration, and Invasion in Renal Cell Carcinoma Cells. Oncol Res. 2017;25(2):157-66.

12. Zhang LG, Zhou XK, Zhou RI, Lv HZ, Li WP. Long non-coding RNA LINC00673 promotes hepatocellular carcinoma progression and metastasis through negatively regulating miR-205. Am J Cancer Res. 2017;7(12):2536-44.

13. Li JH, Zhang SO Oiu XG, Zhang SJ, Zheng SH, Zhang DH. Long non-coding RNA NEAT1 promotes malignant progression of thyroid carcinoma by regulating miRNA-214. Int J Oncol. 2017;50(2):708-16.

14. Zeng J, Du T, Song Y, Gao Y, Li F, Wu R, et al. Knockdown of Long Noncoding RNA CCAT2 Inhibits Cellular Proliferation, Invasion, and Epithelial-Mesenchymal Transition in Glioma Cells. Oncol Res. 2017;25(6):913-21.

15. Lan T, Chang L, Wu L, Yuan Y. Downregulation of ZEB2-AS1 decreased tumor growth and metastasis in hepatocellular carcinoma. Mol Med Rep. 2016;14(5):4606-12.

16. Cui M, Xiao Z, Wang $\mathrm{Y}$, Zheng $\mathrm{M}$, Song $\mathrm{T}$, Cai X, et al. Long noncoding RNA HULC modulates abnormal lipid metabolism in hepatoma cells through an miR-9-mediated RXRA signaling pathway. Cancer Res. 2015;75(5):846-57.

17. Tang J, Zhuo H, Zhang X, Jiang R, Ji J, Deng L, et al. A novel biomarker Linc00974 interacting with KRT19 promotes proliferation and metastasis in hepatocellular carcinoma. Cell Death Dis. 2014;5:e1549.

18. Lian Y, Yang J, Lian Y, Xiao C, Hu X, Xu H. DUXAP8, a pseudogene derived lncRNA, promotes growth of pancreatic carcinoma cells by epigenetically silencing CDKN1A and KLF2. Cancer Commun (Lond). 2018;38(1):64.

19. Huang T, Wang $X$, Yang $X$, Ji J, Wang Q, Yue X, et al. Long Non-Coding RNA DUXAP8 Enhances Renal Cell Carcinoma Progression via Downregulating miR-126. Med Sci Monit. 2018:24·7340-7.

20. Jiang B, Hailong S, Yuan J, Zhao H, Xia W, Zha Z, et al. Identification of oncogenic long noncoding RNA SNHG12 and DUXAP8 in human bladder cancer through a comprehensive profiling analysis. Biomed Pharmacother. 2018;108:500-7.

21. Xu LJ, Yu XJ, Wei B, Hui HX, Sun Y, Dai J, et al. Long non-coding RNA DUXAP8 regulates proliferation and invasion of esophageal squamous cell cancer. Eur Rev Med Pharmacol Sci. 2018;22(9):2646-52

22. Xu X, Xu Y, Shi C, Wang B, Yu X, Zou Y, et al. A genome-wide comprehensively analyses of long noncoding RNA profiling and metastasis associated lncRNAs in renal cell carcinoma. Oncotarget. 2017;8(50):87773-81.

23. Sun $M$, Nie FQ, Zang C, Wang $Y$, Hou J, Wei C, et al. The Pseudogene DUXAP8 Promotes Non-small-cell Lung Cancer Cell Proliferation and Invasion by Epigenetically Silencing EGR1 and RHOB. Mol Ther. 2017;25(3):739-51.

24. Liu W, Zhang Y, Chen M, Shi L, Xu L, Zou X. A genome-wide analysis of long noncoding RNA profile identifies differentially expressed lncRNAs associated with Esophageal cancer. Cancer Med. 2018;7(8):4181-9.

25. Paci P, Colombo T, Farina L. Computational analysis identifies a sponge interaction network between long non-coding RNAs and messenger RNAs in human breast cancer. BMC systems biology. 2014;8:83.

26. Liao Q, Liu C, Yuan X, Kang S, Miao R, Xiao H, et al. Large-scale prediction of long non-coding RNA functions in a coding-non-coding gene co-expression network. Nucleic Acids Res. 2011;39(9):3864-78.

27. Tang Z, Li C, Kang B, Gao G, Li C, Zhang Z. GEPIA: a web server for cancer and normal gene expression profiling and interactive analyses. Nucleic Acids Res. 2017;45(W1):W98-w102.

28. Wang $\mathrm{X}$, Liao $\mathrm{X}$, Yang $\mathrm{C}$, Huang $\mathrm{K}, \mathrm{Yu} \mathrm{T}, \mathrm{Yu} \mathrm{L}$, et al. Identification of prognostic biomarkers for patients with hepatocellular carcinoma after hepatectomy. Oncol Rep. 2019;41(3):1586-602.

29. Zhou M, Zhao H, Wang Z, Cheng L, Yang L, Shi H, et al. Identification and validation of potential prognostic lncRNA biomarkers for predicting survival in patients with multiple myeloma. J Exp Clin Cancer Res. 2015;34:102. 
30. Montojo J, Zuberi K, Rodriguez H, Kazi F, Wright G, Donaldson SL, et al. GeneMANIA Cytoscape plugin: fast gene function predictions on the desktop. Bioinformatics. 2010;26(22):2927.

31. Shannon P, Markiel A, Ozier O, Baliga NS, Wang JT, Ramage D, et al Cytoscape: a software environment for integrated models of biomolecular interaction networks. Genome Research. 2003;13(11):2498.

32. Szklarczyk D, Morris JH, Cook H, Kuhn M, Wyder S, Simonovic M, et al. The STRING database in 2017: quality-controlled protein-protein association networks, made broadly accessible. Nucleic Acids Res. 2017;45(D1):D362-d8.

33. Robinson MD, McCarthy DJ, Smyth GK. edgeR: a Bioconductor package for differential expression analysis of digital gene expression data. Bioinformatics. 2010;26(1):139-40.

34. Huang DW, Sherman BT, Lempicki RA. Bioinformatics enrichment tools: paths toward the comprehensive functional analysis of large gene lists. Nucleic Acids Research. 2009;37(1):1.

35. Huang DW, Sherman BT, Lempicki RA. Systematic and integrative analysis of large gene lists using DAVID bioinformatics resources. Nature Protocols. 2009;4(1):44.

36. Maere S, Heymans K, Kuiper M. BiNGO: a Cytoscape plugin to assess overrepresentation of gene ontology categories in biological networks. Bioinformatics. 2005;21(16):3448-9.

37. Lian Q, Wang S, Zhang G, Wang D, Luo G, Tang J, et al. HCCDB: A Database of Hepatocellular Carcinoma Expression Atlas. Genomics, proteomics \& bioinformatics. 2018;16(4):269-75.

38. Wurmbach E, Chen YB, Khitrov G, Zhang W, Roayaie S, Schwartz M, et al. Genome-wide molecular profiles of HCV-induced dysplasia and hepatocellular carcinoma. Hepatology. 2007;45(4):938-47.

39. Mas VR, Maluf DG, Archer KJ, Yanek K, Kong X, Kulik L, et al. Genes involved in viral carcinogenesis and tumor initiation in hepatitis C virus-induced hepatocellular carcinoma. Molecular medicine (Cambridge, Mass). 2009;15(3-4):85-94

40. Chen X, Fan S, Song E. Noncoding RNAs: New Players in Cancers. Advances in experimental medicine and biology. 2016;927:1-47.

41. Kondo Y, Shinjo K, Katsushima K. Long non-coding RNAs as an epigenetic regulator in human cancers. Cancer Sci. 2017;108(10):1927-33.

42. Li Y, Guo D, Zhao Y, Ren M, Lu G, Wang Y, et al. Long non-coding RNA SNHG5 promotes human hepatocellular carcinoma progression by regulating miR-26a-5p/GSK3beta signal pathway. Cell Death Dis. 2018;9(9):888.

43. Schmitt AM, Chang HY. Long Noncoding RNAs in Cancer Pathways. Cancer Cell. 2016;29(4):452-63.

44. Li X, Liu R, Yang J, Sun L, Zhang L, Jiang Z, et al. The role of long noncoding RNA H19 in gender disparity of cholestatic liver injury in multidrug resistance 2 gene knockout mice. Hepatology. 2017;66(3):869-84.

45. Heubach J, Monsior J, Deenen R, Niegisch G, Szarvas T, Niedworok C, et al. The long noncoding RNA HOTAIR has tissue and cell type-dependent effects on HOX gene expression and phenotype of urothelial cancer cells. Mol Cancer. 2015;14:108.

46. Malakar P, Shilo A, Mogilevsky A, Stein I, Pikarsky E, Nevo Y, et al. Long Noncoding RNA MALAT1 Promotes Hepatocellular Carcinoma Development by SRSF1 Upregulation and mTOR Activation. Cancer Res. 2017;77(5):1155-67.

47. Lin PC, Huang HD, Chang CC, Chang YS, Yen JC, Lee CC, et al. Long noncoding RNA TUG1 is downregulated in non-small cell lung cancer and can regulate CELF1 on binding to PRC2. BMC Cancer. 2016;16:583.

48. Ye K, Wang S, Zhang H, Han H, Ma B, Nan W. Long Noncoding RNA GAS5 Suppresses Cell Growth and Epithelial-Mesenchymal Transition in Osteosarcoma by Regulating the miR-221/ARHI Pathway. J Cell Biochem. 2017;118(12):4772-81.

49. Deng L, Yang SB, Xu FF, Zhang JH. Long noncoding RNA CCAT1 promotes hepatocellular carcinoma progression by functioning as let-7 sponge. J Exp Clin Cancer Res. 2015;34:18.

50. Huarte $M$. The emerging role of lncRNAs in cancer. Nature medicine. 2015;21(11):1253-61.

51. Hu Y, Wang J, Qian J, Kong X, Tang J, Wang Y, et al. Long noncoding RNA GAPLINC regulates CD44-dependent cell invasiveness and associates with poor prognosis of gastric cancer. Cancer Res. 2014;74(23):6890-902.

52. Flockhart RJ, Webster DE, Qu K, Mascarenhas N, Kovalski J, Kretz M, et al. BRAFV600E remodels the melanocyte transcriptome and induces BANCR to regulate melanoma cell migration. Genome Res. 2012;22(6):1006-14.

53. Yuan JH, Yang F, Wang F, Ma JZ, Guo YJ, Tao QF, et al. A long noncoding RNA activated by TGF-beta promotes the invasion-metastasis cascade in hepatocellular carcinoma. Cancer Cell. 2014;25(5):666-81.

54. Ma HW, Xie M, Sun M, Chen TY, Jin RR, Ma TS, et al. The pseudogene derived long noncoding RNA DUXAP8 promotes gastric cancer cell proliferation and migration via epigenetically silencing PLEKHO1 expression. Oncotarget. 2017;8(32):52211-24.

55. Singh P, Singh IN, Mondal SC, Singh L, Garg VK. Platelet-activating factor (PAF)-antagonists of natural origin. Fitoterapia. 2013;84:180-201.

56. Nobili S, Landini I, Giglioni B, Mini E. Pharmacological strategies for overcoming multidrug resistance. Current drug targets. 2006;7(7):861-79.

57. Jin ZL, Yan W, Qu M, Ge CZ, Chen X, Zhang SF. Cinchonine activates endoplasmic reticulum stress-induced apoptosis in human liver cancer cells. Exp Ther Med. 2018;15(6):5046-50

58. Qi Y, Pradipta AR, Li M, Zhao X, Lu L, Fu X, et al. Cinchonine induces apoptosis of HeLa and A549 cells through targeting TRAF6. J Exp Clin Cancer Res. 2017;36(1):35
59. Teng F, Guo M, Liu F, Wang C, Dong J, Zhang L, et al. Treatment with an SLC12A1 antagonist inhibits tumorigenesis in a subset of hepatocellular carcinomas. Oncotarget. 2016;7(33):53571-82.

60. Yarmohammadi H, Wilkins LR, Erinjeri JP, Novak RD, Exner AA, Wu H, et al. Efficiency of combined blocking of aerobic and glycolytic metabolism pathways in treatment of N1-S1 hepatocellular carcinoma in a rat model. J Cancer Res Ther. 2017;13(3):533-7.

61. Riskin WG, Gillings DB, Scarlett JA. Amiprilose hydrochloride for rheumatoid arthritis. Annals of internal medicine. 1989;111(6):455-65.

62. Wang KC, Chang HY. Molecular mechanisms of long noncoding RNAs. Molecular cell. 2011;43(6):904-14.

63. Yue C, Liang C, Li P, Yan L, Zhang D, Xu Y, et al. DUXAP8 a Pan-Cancer Prognostic Marker Involved in the Molecular Regulatory Mechanism in Hepatocellular Carcinoma: A Comprehensive Study Based on Data Mining, Bioinformatics, and in vitro Validation. 2019;12:11637-50.

64. Jiang H, Shi X, Ye G, Xu Y, Xu J, Lu J, et al. Up-regulated long non-coding RNA DUXAP8 promotes cell growth through repressing Krüppel-like factor 2 expression in human hepatocellular carcinoma. Onco Targets Ther. 2019;12:7429-36.

65. Wei F, Yang L, Jiang D. Long noncoding RNA DUXAP8 contributes to the progression of hepatocellular carcinoma via regulating miR-422a/PDK2 axis. 2020;9(7):2480-90.

66. Qu C, Qu Y. Down-regulation of salt-inducible kinase 1 (SIK1) is mediated by RNF2 in hepatocarcinogenesis. Oncotarget. 2017;8(2):3144-55.

67. Belenky M, Prasain J, Kim H, Barnes S. DING, a genistein target in human breast cancer: a protein without a gene. The Journal of nutrition. 2003;133(7 Suppl):2497s-501s 